\title{
Local-in-time existence of strong solutions to a class of compressible non-Newtonian Navier-Stokes equations
}

\author{
Martin Kalousek, Václav Máchał Š́rka Nečasováł
}

December 4, 2020

\begin{abstract}
The aim of this article is to show a local-in-time existence of a strong solution to the generalized compressible Navier-Stokes equation for arbitrarily large initial data. The goal is reached by $L^{p}$-theory for linearized equations which are obtained with help of the Weis multiplier theorem and can be seen as generalization of the work of Shibata and Enomoto [13] (devoted to compressible fluids) to compressible non-Newtonian fluids.
\end{abstract}

Keywords: non-Newtonian fluids, the Weis theorem, $L^{p}$-theory

AMS subject classification: 35A01, 35B65, 35P09, 35Q35

\section{Introduction}

In this paper, we analyze a boundary value problem for Navier-Stokes equations describing the flow of a compressible non-Newtonian fluid that reads

$$
\begin{array}{rlrl}
\partial_{t}(\rho u)+\operatorname{div}(\rho u \otimes u)+\nabla \pi(\rho) & =\operatorname{div} \mathcal{S} & \text { in } Q_{T}, \\
\partial_{t} \rho+\operatorname{div}(\rho u) & =0 & & \text { in } Q_{T}, \\
u(0, \cdot)=u_{0}, \rho(0, \cdot) & =\rho_{0} & & \text { in } \Omega .
\end{array}
$$

Here $\Omega \subset \mathbf{R}^{d}, d \geq 2$ is the domain occupied by the fluid, $T>0$ is the time of evolution and $Q_{T}=(0, T) \times \Omega$. We denote by $u(t, x)$ the fluid velocity, by $\rho(t, x)$ the fluid density and by $\mathcal{S}$ the viscous stress. We assume the periodic boundary condition, i.e., $\Omega$ is a torus

$$
\Omega=\left(\left.[-1,1]\right|_{\{-1,1\}}\right)^{d} .
$$

We restrict ourselves to the constitutive relation

$$
\mathcal{S}=2 \mu\left(\left|\mathbb{D}^{D} u\right|^{2}\right) \mathbb{D}^{D} u+\lambda(\operatorname{div} u) \operatorname{div} u \mathbb{I}_{d}
$$

where $\mu$ and $\lambda$ are viscosity coefficients whose properties will be specified later.

*kalousek@math.cas.cz, Institute of Mathematics, Czech Academy of Sciences, Žitná 25, 115 67 Praha 1, Czech Republic

${ }^{\dagger}$ macha@math.cas.cz, Institute of Mathematics, Czech Academy of Sciences, Žitná 25, 11567 Praha 1, Czech Republic

${ }_{\ddagger}^{\ddagger}$ matus@math.cas.cz, Institute of Mathematics, Czech Academy of Sciences, Žitná 25, 11567 Praha 1, Czech Republic 


\subsection{Discussion and main result}

The system with $\mu \equiv \mu_{0} \in \mathbb{R}$ and $\lambda \equiv \lambda_{0} \in \mathbb{R}$ has been extensively studied. The mathematical analysis of compressible viscous fluids goes back to 1950s. The first result concerning uniqueness was given by Graffi [15] and Serrin [32]. A local in time existence in Hölder continuous spaces was proven by Nash [29], Itaya [16, 17] and Vol'pert and Hudjaev [38]. In Sobolev-Slobodetskii space the local existence was shown by Solonnikov [34]. The local-in-time existence and global-in-time existence for small data in Hilbert space traces back to Valli [36. The optimal regularity of local in time solutions was obtained by Charve and Danchin [9. The global solution with the initial data close to a rest state was shown by Matsumura-Nishida [27, 26] in the whole space and the exterior domain. The case of bounded domain was treated in work of Valli, Zajaczkowski [37].

The global-in-time existence of a weak solution is much more recent and goes back to the half of of 1990s. The existence is known due to nowadays standard Feireisl - Lions theory - we refer to 14] and to [21.

However, there is an unsatisfactory number of articles for the system with a general $\mu$ and $\lambda$. In particular, Mamontov [24], 25] considered an exponentially growing viscosity and isothermal pressure. For such case the global existence of a weak solution was shown. Recently, Abbatiello, Feireisl and Novotný in [1] provide a proof of the existence of so-called dissipative solution - a notion of solution which is 'weaker' than a weak solution. The existence of weak solution is still unproven. The main obstacle seems to be the lack of compactness of velocity $u$ which is caused by an insufficient control of $\partial_{t} u$ in (and near) vacuum regions.

Let us mention that such type of problem was already studied in the incompressible case by Ladyzhenskaya [20] and then extensively studied by group of J. Nečas [6, [5, 23]. Further, basic properties of weak or measure-valued solution of incompressible non-Newtonian fluids are described in [22, 4. Concerning the compressible case the first attempt to solve this problem can be found in work of Nečasová, Novotný [28] where the existence of measure-valued solution was shown.

The concept of the maximal regularity is classical and the main achievements for abstract theory go back to the work of Ladyzhenskaya, Uraltzeva, Solonnikov [19], Da-Prato, Grisvard [11, Amann [2] and Prüss [30]. The notion of $\mathcal{R}$-sectoriality was introduced by Clement and Prüss see [10]. The fundamental result by Weis [39] about equivalency of description for the maximal regularity in terms of vector-valued Fourier multipliers and $\mathcal{R}$ sectoriality was a breakthrough for the applications.

The Weis multiplier theorem allows to deduce the $L^{p}$ maximal regularity to problems connected with the fluid dynamics. We refer to [12] and [18] for the comprehensible decription of the method. The maximal $L^{p}$ regularity result for incompressible fluids can be found in work by Shibata, Shimizu [33], for the non-Newtonian incompressible situation it was established in work by Bothe and Prüss [7]. Maximal regularity for the compressible case was shown by Shibata, Enomoto[13].

The goal of this paper is to use the Weis multiplier theorem to show the short-time existence of a strong solution in $L^{p}$ setting for the non-Newtonian compressible fluid.

Let us introduce our main result.

Theorem 1.1. Let $\mu \in \mathcal{C}^{3}([0, \infty))$ and $\lambda \in \mathcal{C}^{2}(\mathbf{R})$ satisfy $\mu(s)+2 \mu^{\prime}(s) s>0$ for all $s \geq 0$ and $\lambda(r)+\lambda^{\prime}(r) r>0$ for all $r \in \mathbf{R}$. Let, moreover, $\pi \in \mathcal{C}^{2}([0, \infty)), q>d$ and $p \in(1, \infty)$ be given. Then for every $u_{0} \in W^{2, q}(\Omega)$ and $\rho_{0} \in W^{1, q}(\Omega), \frac{1}{\rho_{0}} \in L^{\infty}(\Omega)$ there is $T>0$ such that there exists

$$
(\rho, u) \in L^{p}\left(0, T ; W^{1, q}(\Omega)\right) \times L^{p}\left(0, T ; W^{2, q}(\Omega)\right)
$$

with

$$
\left(\partial_{t} \rho, \partial_{t} u\right) \in L^{p}\left(0, T ; W^{1, q}(\Omega)\right) \times L^{p}\left(0, T ; L^{q}(\Omega)\right)
$$


which satisfies (11).

The proof of the main result relies on several steps. The first step is the use of Lagrange coordinates to get rid of the convective term.

Secondly, we linearize the resulting system and we show $L^{p}-L^{q}$ regularity property of a solution. This is discussed in Section 3 and it is obtained by means of the Weis multiplier theorem.

The sufficient regularity of the velocity $u$ allows to deduce appropriate bounds and one may use the Banach fix-point argument to deduce the existence of a strong solution assuming the time $T>0$ is small enough. This is described in Section 2 .

The remaining part of this introductory section is devoted to necessary preliminary statements.

\subsection{Notation}

We denote by $\mathbb{I}_{d}$ the $d \times d$ identity matrix. For the $d \times d$-matrix valued mapping $G \operatorname{sym} G$ denotes the symmetric part of $G$, i.e., $\operatorname{sym} G=\frac{1}{2}\left(G+G^{\top}\right)$ and $G^{D}$ is the traceless part of $G$, i.e., $G^{D}=G-\frac{1}{d} \operatorname{tr} G \mathbb{I}_{d}$. Let $u$ be a mapping with values in $\mathbf{R}^{d}$ then $\mathbb{D} u=\operatorname{sym}(\nabla u)$ is the symmetric part of the gradient and $\mathbb{D}^{D} u$ stands for the traceless part of $\mathbb{D} u$. For purposes of this paper we denote by $\mathcal{V}^{p, q}\left(Q_{T}\right)$ the following function space

$$
\mathcal{V}^{p, q}\left(Q_{T}\right)=L^{p}\left(0, T ; W^{2, q}(\Omega)\right) \cap W^{1, p}\left(0, T ; L^{q}(\Omega)\right)
$$

with the norm $\|\cdot\|_{V^{p, q}\left(Q_{T}\right)}=\|\cdot\|_{L^{p}\left(0, T ; W^{2, q}(\Omega)\right)}+\|\cdot\|_{W^{1, p}\left(0, T ; L^{q}(\Omega)\right)}$. Starting with definition (2), using the fact that $\operatorname{tr} \mathbb{D}^{D} u=0$ and the symmetry of $\mathbb{D}^{D} u$ we have

$$
\begin{aligned}
(\operatorname{div} \mathcal{S})_{j}= & 2 \mu\left(\left|\mathbb{D}^{D} u\right|^{2}\right) \sum_{l=1}^{d} \partial_{k}\left(\left(\mathbb{D}^{D} u\right)_{j l}-\frac{1}{d} \delta_{j l} \operatorname{div} u\right) \\
& +4 \mu^{\prime}\left(\left|\mathbb{D}^{D} u\right|^{2}\right) \sum_{k, l, m=1}^{d}\left(\mathbb{D}^{D} u\right)_{j k}\left(\mathbb{D}^{D} u\right)_{l m} \partial_{k}\left((\mathbb{D} u)_{l m}-\frac{1}{d} \delta_{l m} \operatorname{div} u\right) \\
& +\sum_{l=1}^{d} \delta_{j l}\left(\lambda(\operatorname{div} u)+\lambda^{\prime}(\operatorname{div} u) \operatorname{div} u\right) \partial_{l} \operatorname{div} u \\
= & \mu\left(\left|\mathbb{D}^{D} u\right|^{2}\right) \sum_{l=1}^{d}\left(\partial_{l}^{2} u_{j}+\partial_{j} \partial_{l} u_{l}\right)+2 \mu^{\prime}\left(\left|\mathbb{D}^{D} u\right|^{2}\right) \sum_{k, l, m=1}^{d}\left(\mathbb{D}^{D} u\right)_{j k}\left(\mathbb{D}^{D} u\right)_{l m}\left(\partial_{k} \partial_{m} u_{l}+\partial_{k} \partial_{l} u_{m}\right) \\
& -\frac{2}{d} \mu\left(\left|\mathbb{D}^{D} u\right|^{2}\right) \partial_{j} \operatorname{div} u+\left(\lambda(\operatorname{div} u)+\lambda^{\prime}(\operatorname{div} u) \operatorname{div} u\right) \partial_{j} \operatorname{div} u \\
= & \mu\left(\left|\mathbb{D}^{D} u\right|^{2}\right) \sum_{k=1}^{d}\left(\partial_{l}^{2} u_{j}+\partial_{j} \partial_{l} u_{j}\right)+4 \mu^{\prime}\left(\left|\mathbb{D}^{D} u\right|^{2}\right) \sum_{k, l, m=1}^{d}\left(\mathbb{D}^{D} u\right)_{j l}\left(\mathbb{D}^{D} u\right)_{k m} \partial_{l} \partial_{m} u_{k} \\
& -\frac{2}{d} \mu\left(\left|\mathbb{D}^{D} u\right|^{2}\right) \partial_{j} \operatorname{div} u+\left(\lambda(\operatorname{div} u)+\lambda^{\prime}(\operatorname{div} u) \operatorname{div} u\right) \partial_{j} \operatorname{div} u
\end{aligned}
$$

Hence we deduce that

$$
(\operatorname{div} \mathcal{S})_{j}=\sum_{k, l, m=1}^{d} a_{j k}^{l m}(\mathbb{D} u) \partial_{m} \partial_{l} u_{k}
$$


where

$$
\begin{aligned}
a_{j k}^{l m}(\mathbb{D} u)= & \mu\left(\left|\mathbb{D}^{D} u\right|^{2}\right)\left(\delta_{j k} \delta_{l m}+\delta_{j m} \delta_{k l}\right)+4 \mu^{\prime}\left(\left|\mathbb{D}^{D} u\right|^{2}\right)\left(\mathbb{D}^{D} u\right)_{j l}\left(\mathbb{D}^{D} u\right)_{k m} \\
& +\left(\lambda(\operatorname{div} u)+\lambda^{\prime}(\operatorname{div} u) \operatorname{div} u-\frac{2}{d} \mu\left(\left|\mathbb{D}^{D} u\right|^{2}\right)\right) \delta_{k m} \delta_{j l} .
\end{aligned}
$$

We note that $a_{j k}^{l m}$ possesses the following symmetries

$$
a_{j k}^{l m}=a_{k j}^{l m}=a_{l m}^{j k}=a_{l k}^{j m}=a_{j m}^{l k} \text { for all } j, k, l, m=1, \ldots, d .
$$

We define for a given $u \in C^{1}(\bar{\Omega})^{d}$ the quasilinear differential operator $\mathcal{A}$ as

$$
\mathcal{A}(\mathbb{D} u)(\mathbb{D} v)=\sum_{l, m=1}^{d} a_{j k}^{l m}(\mathbb{D} u) \partial_{l} \partial_{m} v
$$

whose ellipticity is ensured by certain identities satisfied by $\mu, \lambda$ and their derivatives as we now show. Considering $\xi \in \mathbf{R}_{\text {sym }}^{d \times d}$ we get

$$
\begin{aligned}
a_{j k}^{l m} \xi_{j l} \xi_{k m}= & \mu\left(\left|\mathbb{D}^{D} u\right|^{2}\right)\left(\xi_{j l}^{2}+\xi_{k m}^{2}\right)+4 \mu^{\prime}\left(\left|\mathbb{D}^{D} u\right|^{2}\right)\left(\mathbb{D}^{D} u\right)_{j l} \xi_{j l}\left(\mathbb{D}^{D} u\right)_{k m} \xi_{k m} \\
& +\left(\lambda(\operatorname{div} u)+\lambda^{\prime}(\operatorname{div} u) \operatorname{div} u-\frac{2}{d} \mu\left(\left|\mathbb{D}^{D} u\right|^{2}\right)\right) \xi_{j j} \xi_{l l} .
\end{aligned}
$$

Hence we obtain

$$
\begin{aligned}
\sum_{j, k, l, m=1}^{d} a_{j k}^{l m} \xi_{j l} \xi_{k m}= & 2 \mu\left(\left|\mathbb{D}^{D} u\right|^{2}\right)|\xi|^{2}+4 \mu^{\prime}\left(\left|\mathbb{D}^{D} u\right|^{2}\right)\left|\mathbb{D}^{D} u \cdot \xi\right|^{2} \\
& +\left(\lambda(\operatorname{div} u)+\lambda^{\prime}(\operatorname{div} u) \operatorname{div} u-\frac{2}{d} \mu\left(\left|\mathbb{D}^{D} u\right|^{2}\right)\right)(\operatorname{tr} \xi)^{2} .
\end{aligned}
$$

Applying the decomposition $\xi=\xi^{D}+\frac{\operatorname{tr} \xi}{d} \mathbb{I}_{d}$, in particular the fact that $\xi^{D} \cdot \mathbb{I}_{d}=0$, we arrive at

$$
\sum_{j, k, l, m=1}^{d} a_{j k}^{l m} \xi_{j l} \xi_{k m}=2 \mu\left(\left|\mathbb{D}^{D} u\right|^{2}\right)\left|\xi^{d}\right|^{2}+4 \mu^{\prime}\left(\left|\mathbb{D}^{D} u\right|^{2}\right)\left|\mathbb{D}^{D} u \cdot \xi^{d}\right|^{2}+\left(\lambda(\operatorname{div} u)+\lambda^{\prime}(\operatorname{div} u) \operatorname{div} u\right)(\operatorname{tr} \xi)^{2}
$$

We deal with the notion of strong ellipticity of the operator $\mathcal{A}(\mathbb{D} u)$ which means that there exists $C_{e l}(\mathbb{D} u)>0$ such that

$$
\sum_{j, k, l, m=1}^{d} a_{j k}^{l m} \xi_{j l} \xi_{k m} \geq C_{e l}(\mathbb{D} u)|\xi|^{2} \text { holds for all } \xi \in \mathbf{R}^{d \times d} .
$$

Let investigate necessary conditions on $\mu$ and $\lambda$ related to the strong ellipticity of $\mathcal{A}(\mathbb{D} u)$. We first choose $g \in \mathbf{R}^{d}$ as an eigenvector of $\mathbb{D}^{D} u$ and $h \in \mathbf{R}^{d}$ being perpendicular to $g$. We consider the matrix $(\xi)_{j k}=\frac{1}{2}\left(g_{j} h_{k}-g_{k} h_{j}\right), j, k=1, \ldots d$ and we obtain from (77) combined with (8)

$$
2 \mu\left(\left|\mathbb{D}^{D} u\right|^{2}\right)|\xi|^{2} \geq C_{e l}(\mathbb{D} u)|\xi|^{2}
$$

as $\xi$ is obviously traceless and $\xi^{D}=\xi$ consequently. Hence one concludes that

$$
2 \mu(s) \geq C_{e l}(\mathbb{D} u) \text { for all } s \in\left[0,\left\|\mathbb{D}^{D} u\right\|_{L^{\infty}(\Omega)}^{2}\right] .
$$


As $u \in C^{1}(\bar{\Omega})^{d}$ can be chosen arbitrarily, we get

$$
\mu(s)>0 \text { for any } s \geq 0 \text {. }
$$

In order to get the next condition involving $\mu$, we set $\xi=\mathbb{D}^{D} u$ in (8) , which together with (7) imply

$$
2 \mu\left(\left|\mathbb{D}^{D} u\right|^{2}\right)\left|\mathbb{D}^{D} u\right|^{2}+4 \mu^{\prime}\left(\left|\mathbb{D}^{D} u\right|^{2}\right)\left|\mathbb{D}^{D} u\right|^{2}\left|\mathbb{D}^{D} u\right|^{2} \geq C_{e l}(u)\left|\mathbb{D}^{D} u\right|^{2}
$$

Hence one concludes that

$$
2 \mu(s)+4 \mu^{\prime}(s) s \geq C_{e l}(\mathbb{D} u), \text { for any } s \in\left[0,\left\|\mathbb{D}^{D} u\right\|_{L^{\infty}(\Omega)}^{2}\right]
$$

and finally

$$
\mu(s)+2 \mu^{\prime}(s) s>0 \text { for any } s \geq 0
$$

by repeating the arguments leading to (9). To determine a condition on $\lambda$ we choose $\xi=\mathbb{I}_{d}$ in (8)). It follows that $\left(\mathbb{I}_{d}\right)^{D}=0$ and we obtain from (7) that

$$
\left(\lambda(\operatorname{div} u)+\lambda^{\prime}(\operatorname{div} u) \operatorname{div} u\right) d^{2} \geq C_{e l}(\mathbb{D} u) d .
$$

Repeating the arguments leading to (9), (10) respectively, we get

$$
\lambda(r)+\lambda^{\prime}(r) r \geq \frac{C_{e l}(u)}{d} \text { for all } r \in\left[-\|\operatorname{div} u\|_{L^{\infty}(\Omega)},\|\operatorname{div} u\|_{L^{\infty}(\Omega)}\right]
$$

and then

$$
\lambda(r)+\lambda^{\prime}(r) r>0 \text { for all } r \in \mathbf{R} .
$$

We note that conditions (9), (10) and (11) are also sufficient for the ellipticity of $\mathcal{A}(\mathbb{D} u)$. If $\mu^{\prime}\left(\left|\mathbb{D}^{D} u\right|^{2}\right) \geq 0$ these conditions ensure that

$$
\sum_{j, k, l, m=1}^{d} a_{j k}^{l m} \xi_{j l} \xi_{k m} \geq 2 \mu\left(\left|\mathbb{D}^{D} u\right|^{2}\right)\left|\xi^{d}\right|^{2}+\left(\lambda(\operatorname{div} u)+\lambda^{\prime}(\operatorname{div} u) \operatorname{div} u\right)(\operatorname{tr} \xi)^{2} .
$$

From the continuity of $\mu$ and the function $r \mapsto \lambda(r)+\lambda(r)^{\prime} r$ and from the assumption $u \in C^{1}(\bar{\Omega})^{d}$ we conclude the existence of $s_{\min } \in\left[0,\left\|\mathbb{D}^{D} u\right\|_{L^{\infty}(\Omega)}^{2}\right]$ and $r_{\min } \in\left[-\|\operatorname{div} u\|_{L^{\infty}},\|\operatorname{div} u\|_{L^{\infty}}\right]$ such that $\mu(s) \geq \mu\left(s_{\text {min }}\right)>0$ for all $s \in\left[0,\left\|\mathbb{D}^{D} u\right\|_{L^{\infty}(\Omega)}^{2}\right]$ and $\lambda(r)+\lambda^{\prime}(r) r \geq \lambda\left(r_{\text {min }}\right)+\lambda^{\prime}\left(r_{\text {min }}\right) r_{\text {min }}>0$ for all $r \in\left[-\|\operatorname{div} u\|_{L^{\infty}},\|\operatorname{div} u\|_{L^{\infty}}\right]$. It follows from (12) that

$$
\sum_{j, k, l, m=1}^{d} a_{j k}^{l m} \xi_{j l} \xi_{k m} \geq 2 \mu\left(s_{\text {min }}\right)\left|\xi^{d}\right|^{2}+\left(\lambda\left(r_{\text {min }}\right)+\lambda^{\prime}\left(r_{\text {min }}\right) r_{\text {min }}\right)(\operatorname{tr} \xi)^{2} .
$$

Moreover, since one can easily check that the mapping $\xi \mapsto \sqrt{\left|\xi^{D}\right|^{2}+(\operatorname{tr} \xi)^{2}}$ is an equivalent norm on $\mathbf{R}^{d \times d}$, the ellipticity of $\mathcal{A}(u)$ follows from (13) by setting $C_{e l}=\min \left\{2 \mu\left(s_{\min }\right), \lambda\left(r_{\min }\right)+\right.$ $\left.\left.\lambda^{\prime}\left(r_{\min }\right) r_{\min }\right)\right\}$.

If $\mu^{\prime}\left(\left|\mathbb{D}^{D} u\right|^{2}\right)<0$ one applies the Cauchy-Schwarz inequality in (17) to obtain

$$
\begin{aligned}
& \sum_{j, k, l, m=1}^{d} a_{j k}^{l m} \xi_{j l} \xi_{k m} \geq \\
& \quad 2 \mu\left(\left|\mathbb{D}^{D} u\right|^{2}\right)\left|\xi^{d}\right|^{2}+4 \mu^{\prime}\left(\left|\mathbb{D}^{D} u\right|^{2}\right)\left|\mathbb{D}^{D} u\right|^{2}\left|\xi^{d}\right|^{2}+\left(\lambda(\operatorname{div} u)+\lambda^{\prime}(\operatorname{div} u) \operatorname{div} u\right)(\operatorname{tr} \xi)^{2} .
\end{aligned}
$$

The continuity of $s \mapsto \mu(s)+2 \mu^{\prime}(s) s$ and the assumption $u \in C^{1}(\bar{\Omega})^{d}$ imply the existence of $\tilde{s}_{\text {min }} \in\left[0,\left\|\mathbb{D}^{D} u\right\|_{L^{\infty}(\Omega)}^{2}\right]$ such that $\mu(s)+2 \mu^{\prime}(s) s \geq \mu\left(\tilde{s}_{\text {min }}\right)+2 \mu^{\prime}\left(\tilde{s}_{\text {min }}\right) \tilde{s}_{\text {min }}>0$ for all $s \in$ $\left[0,\left\|\mathbb{D}^{D} u\right\|_{L^{\infty}(\Omega)}^{2}\right]$. Going back to (14) the ellipticity of $\mathcal{A}(\mathbb{D} u)$ follows by setting $C_{e l}=\min \left\{\mu\left(\tilde{s}_{\text {min }}\right)+\right.$ $\left.2 \mu^{\prime}\left(\tilde{s}_{\text {min }}\right) \tilde{s}_{\text {min }}, \lambda\left(r_{\text {min }}\right)+\lambda^{\prime}\left(r_{\text {min }}\right) r_{\text {min }}\right\}$. 


\section{Local-in-time well posedness of the compressible Navier- Stokes}

In this section we present a proof of Theorem 1.1. First, we transform system (11) into Lagrangian coordinates. Then a linearization of the transformed system is derived. Next, employing Theorem 3.1 we show that the solution operator to the linearized system is a contraction on a suitably chosen function space. Finally, by the Banach fixed point theorem we conclude the local-in-time well posedness of the transformed system and of (1) accordingly.

\subsection{System in Lagrangian coordinates}

We define the Lagrangian coordinates as

$$
X_{u}(t, y)=y+\int_{0}^{t} u\left(s, X_{u}(s, y)\right) \mathrm{d} s .
$$

A general function $f(t, x): \Omega \mapsto \mathbb{R}$ fulfills

$$
\begin{aligned}
\partial_{t} f\left(t, X_{u}(t, y)\right) & =\partial_{1} f\left(t, X_{u}(t, y)\right)+\nabla_{x} f\left(t, X_{u}(t, y)\right) \cdot u \\
\nabla_{y} f\left(t, X_{u}(t, y)\right) & =\nabla_{x} f\left(t, X_{u}(t, y)\right) \nabla_{y} X_{u}(t, y) \\
\nabla_{x} f & =\nabla_{y} f\left(\nabla_{y} X_{u}\right)^{-1} .
\end{aligned}
$$

The Jacobi matrix of the transformation $X_{u}$ is $\mathbb{I}_{d}+\int_{0}^{t} \nabla_{y} u\left(s, X_{u}(s, y)\right) \mathrm{d} s$. We tacitly assume the invertibility of this matrix, which is ensured if

$$
\sup _{t \in(0, T)}\left\|\int_{0}^{t} \nabla_{y} u(s, \cdot) \mathrm{d} s\right\|_{L^{\infty}(\Omega)}<\sigma
$$

for some small number $\sigma$. Since we will work with functions that possess Lipschitz regularity with respect to space variables, the latter condition is fulfilled if $T$ is chosen suitably small. In what follows we use the notation $E_{u}:=\mathbb{I}_{d}-\left(\nabla X_{u}\right)^{-1}$. Let us note that we get from (15)

$$
E_{u}(t, y)=W\left(\int_{0}^{t} \nabla u\left(s, X_{u}(s, y)\right) \mathrm{d} s\right),
$$

where $W$ is a $d \times d$-matrix valued mapping that is smooth with respect to matrices $B \in \mathbf{R}^{d \times d}$ with $|B|<2 \sigma$ and $W(0)=0$. We define $\tilde{\varrho}(t, y)=\varrho(t, X(t, y))$ and $\tilde{u}(t, y)=u(t, X(t, y))$ and we rewrite (11) as

$$
\begin{aligned}
\partial_{t} \tilde{\varrho}+\tilde{\varrho} \operatorname{div}_{y} \tilde{u} & =G(\tilde{\varrho}, \tilde{u}) \\
\tilde{\varrho} \partial_{t} \tilde{u}+\nabla_{y} \pi(\tilde{\varrho})-\operatorname{div}_{y} \mathcal{S}\left(D_{y} \tilde{u}\right) & =F(\tilde{\varrho}, \tilde{u})
\end{aligned}
$$

where

$$
\begin{aligned}
G(\tilde{\varrho}, \tilde{u}) & =-\nabla \tilde{u} \cdot E_{\tilde{u}} \tilde{\varrho} \\
F(\tilde{\varrho}, \tilde{u}) & =\nabla_{y} \pi(\tilde{\varrho}) E_{\tilde{u}}+\operatorname{div}_{y} \tilde{\mathcal{S}}(\tilde{u})-\operatorname{div}_{y} \mathcal{S}\left(\mathbb{D}_{y}^{D} \tilde{u}\right)-\operatorname{tr} \tilde{\mathcal{S}}(\tilde{u}) E_{\tilde{u}} \\
\mathcal{S}\left(\mathbb{D}_{y}^{D} \tilde{u}\right) & =\mu\left(\left|\mathbb{D}_{y}^{D} \tilde{u}\right|^{2}\right) \mathbb{D}_{y}^{D} \tilde{u}+\lambda\left(\operatorname{div}_{y} \tilde{u}\right) \operatorname{div}_{y} \tilde{u} \mathbb{I}_{d} \\
\tilde{\mathcal{S}}(\tilde{u}) & =\mu\left(|\mathcal{G}(\tilde{u})|^{2}\right) \mathcal{G}(\tilde{u})+\lambda(\mathcal{D}(\tilde{u})) \mathcal{D}(\tilde{u}) \mathbb{I}_{d} \\
\mathcal{G}(\tilde{u}) & =\mathbb{D}_{y}^{D} \tilde{u}-\operatorname{sym}\left(\nabla_{y} \tilde{u} E_{\tilde{u}}\right)^{D}, \mathcal{D}(\tilde{u})=\operatorname{div}_{y} \tilde{u}-\nabla_{y} \tilde{u} \cdot E_{\tilde{u}}
\end{aligned}
$$




\subsection{Linearized system}

Let $\tilde{\varrho}=\varrho_{*}+\theta_{0}+\theta$ where $\varrho_{*}$ is a constant and $\theta_{0}=\varrho_{0}-\varrho_{*}$ is independent of $t$. Recall both $\varrho_{*}$ and $\theta_{0}$ are given. Consequently, (18) becomes (recall (6) )

$$
\begin{aligned}
\partial_{t} \theta+\left(\varrho_{*}+\theta_{0}\right) \operatorname{div}_{y}\left(\tilde{u}-u_{0}\right) & =\mathcal{G}(\theta, \tilde{u}) \\
\left(\varrho_{*}+\theta_{0}\right) \partial_{t} \tilde{u}-\mathcal{A}\left(\mathbb{D}_{y} u_{0}\right)\left(\mathbb{D}_{y}\left(\tilde{u}-u_{0}\right)\right)+\pi^{\prime}\left(\varrho_{*}+\theta_{0}\right) \nabla_{y} \theta & =\mathcal{F}(\theta, \tilde{u})
\end{aligned}
$$

where

$$
\begin{aligned}
\mathcal{G}(\theta, \tilde{u})= & G\left(\varrho_{*}+\theta_{0}+\theta, \tilde{u}\right)-\theta \operatorname{div}_{y} \tilde{u}-\left(\rho_{*}+\theta_{0}\right) \operatorname{div}_{y} u_{0} \\
\mathcal{F}(\theta, \tilde{u})= & F\left(\varrho_{*}+\theta_{0}+\theta, \tilde{u}\right)-\theta \partial_{t} \tilde{u}+\operatorname{div}_{y} \mathcal{S}\left(\mathbb{D}_{y}^{D} \tilde{u}\right)-\mathcal{A}\left(\mathbb{D}_{y} u_{0}\right)\left(\mathbb{D}_{y}\left(\tilde{u}-u_{0}\right)\right)+\pi^{\prime}\left(\varrho_{*}+\theta_{0}\right) \nabla_{y} \theta_{0} \\
& +\left(\pi^{\prime}\left(\varrho_{*}+\theta_{0}+\theta\right)-\pi^{\prime}\left(\varrho_{*}+\theta_{0}\right)\right) \nabla_{y}\left(\theta_{0}+\theta\right)
\end{aligned}
$$

and the system is equipped with the initial conditions $\theta(0, \cdot)=0, \tilde{u}(0, \cdot)=u_{0}$. In order to shorten the notation we omit the subscript $y$ in the rest of this section.

\subsection{The fix-point argument}

We define

$$
\begin{gathered}
H_{T, M}=\left\{(\vartheta, w) \in W^{1, p}\left(0, T ; W^{1, q}(\Omega)\right) \times \mathcal{V}^{p, q}\left(Q_{T}\right): \vartheta(0)=0, w(0)=u_{0},\right. \\
\left.[\vartheta, w]_{T, M}=\|\vartheta\|_{W^{1, p}\left(0, T ; W^{1, q}(\Omega)\right)}+\left\|w-u_{0}\right\|_{\mathcal{V}^{p, q}} \leq M\right\}
\end{gathered}
$$

and an operator $\Phi$ as

$$
\Phi(\vartheta, w)=(\theta, \tilde{u})
$$

where $\theta \in W^{1, p}\left(0, T ; W^{1, q}(\Omega)\right), \tilde{u} \in L^{p}\left(0, T ; W^{2, q}(\Omega)^{d}\right) \cap W^{1, p}\left(0, T ; L^{q}(\Omega)^{d}\right)$ is a solution to

$$
\begin{gathered}
\partial_{t} \theta+\left(\varrho_{*}+\theta_{0}\right) \operatorname{div}\left(\tilde{u}-u_{0}\right)=\mathcal{G}(\vartheta, w) \\
\left(\varrho_{*}+\theta_{0}\right) \partial_{t} \tilde{u}-\mathcal{A}\left(\mathbb{D} u_{0}\right)\left(\mathbb{D}\left(\tilde{u}-u_{0}\right)\right)+\pi^{\prime}\left(\varrho_{*}+\theta_{0}\right) \nabla \theta=\mathcal{F}(\vartheta, w) \\
\theta(0)=0, \tilde{u}(0)=u_{0} .
\end{gathered}
$$

In order to apply the Banach fixed-point theorem, we need to justify that for certain values of $T$ and $M$ the operator $\Phi$ is a contraction on $H_{T, M}$. Next we introduce several estimates. We recall the embedding inequality

$$
\sup _{t \in[0, T]}\|u\|_{W^{1, \infty}(\Omega)} \leq c\left(\|u\|_{L^{p}\left(0, T ; W^{2, q}(\Omega)\right)}^{p}+\|u\|_{W^{1, p}\left(0, T ; L^{q}(\Omega)\right)}^{p}\right)^{\frac{1}{p}},
$$

see [35, p. 10] for details, valid for $u \in \mathcal{V}^{p, q}\left(Q_{T}\right), p \in(1, \infty)$ and $q>d$. For an arbitrary $w \in B_{M}=\left\{v \in \mathcal{V}^{p, q}\left(Q_{T}\right): v(0)=u_{0},\left\|v-u_{0}\right\|_{\mathcal{V}^{p, q}\left(Q_{T}\right)} \leq M\right\}$ it follows that

$$
\|w\|_{\left.\mathcal{V}^{p, q}\left(Q_{T}\right)\right)} \in\left[T^{\frac{1}{p}}\left\|u_{0}\right\|_{W^{2, q(\Omega)}}-M, T^{\frac{1}{p}}\left\|u_{0}\right\|_{W^{2, q}(\Omega)}+M\right] .
$$

Accordingly, we have $\| w_{\mathcal{V}^{p, q}\left(Q_{T}\right)} \leq M+L T^{\frac{1}{p}}$ where

$$
L=\left\|\theta_{0}\right\|_{W^{1, q}(\Omega)}+\left\|u_{0}\right\|_{W^{2, q}(\Omega)} .
$$


Employing (21) we deduce that

$$
\left\|\mathbb{D}^{D} w\right\|_{L^{\infty}\left(0, T ; L^{\infty}(\Omega)\right)}+\|\operatorname{div} w\|_{L^{\infty}\left(0, T ; L^{\infty}(\Omega)\right)} \leq c\|w\|_{\mathcal{V}^{p, q}\left(Q_{T}\right)} \leq c\left(M+L T^{\frac{1}{p}}\right) .
$$

By the Sobolev embedding theorem and the Hölder inequality we infer

$$
\int_{0}^{T}\|\nabla w(s \cdot) \mathrm{d} s\|_{L^{\infty}(\Omega)} \leq \int_{0}^{T}\|w(s, \cdot)\|_{W^{2, q}(\Omega)} \mathrm{d} s \leq c T^{\frac{1}{p^{\prime}}}\|w\|_{L^{p}\left(0, T ; W^{2, q}(\Omega)\right)} .
$$

Hence the condition (16) is fulfilled for any $w \in H_{T, M}$ assuming $T$ is suitably small. Moreover, it follows from (17) and the paragraph below it that

$$
\left\|E_{w}(t)\right\|_{W^{k, q}(\Omega)} \leq c\left\|\int_{0}^{t} \nabla w \mathrm{~d} s\right\|_{W^{k, q}(\Omega)} \leq c \int_{0}^{t}\|w\|_{W^{k+1, q}(\Omega)} \mathrm{d} s, \quad k=0,1 .
$$

The Sobolev embedding then yields (since $q>d$ )

$$
\left\|E_{w}(t)\right\|_{L^{\infty}\left(0, T ; L^{\infty}(\Omega)\right)} \leq c T^{\frac{1}{p^{\prime}}}\|w\|_{L^{p}\left(0, T ; W^{2, q}(\Omega)\right)}
$$

and we use Hölder inequality to deduce

$$
\left\|E_{w}\right\|_{L^{p}\left(0, T ; W^{1, q}(\Omega)\right)} \leq c\left(\int_{0}^{T} t^{\frac{p}{p^{\prime}}}\|\nabla w\|_{L^{p}\left(0, T ; W^{1, q}(\Omega)\right)}^{p} \mathrm{~d} t\right)^{\frac{1}{p}} \leq c T\|w\|_{L^{p}\left(0, T ; W^{2, q}(\Omega)\right)} .
$$

By (23) and (21) we infer

$$
\begin{aligned}
\|\mathcal{G}(w)\|_{L^{\infty}\left(0, T ; L^{\infty}(\Omega)\right)} & \leq c\|\nabla w\|_{L^{\infty}\left(0, T ; L^{\infty}(\Omega)\right)}\left(1+\left\|E_{w}\right\|_{L^{\infty}\left(0, T ; L^{\infty}(\Omega)\right)}\right) \\
& \leq c\|w\|_{\mathcal{V}^{p, q}\left(Q_{T}\right)}\left(1+T^{\frac{1}{p^{\prime}}}\|w\|_{L^{p}\left(0, T ; W^{2, q}(\Omega)\right)}\right)
\end{aligned}
$$

and thus

$$
\|\mathcal{G}(w)\|_{L^{\infty}\left(0, T ; L^{\infty}(\Omega)\right)} \leq c\left(M+L T^{\frac{1}{p}}\right)\left(1+T^{\frac{1}{p^{\prime}}}\left(M+L T^{\frac{1}{p}}\right)\right)
$$

due to (21). One similarly deduces

$$
\|\mathcal{D}(w)\|_{L^{\infty}\left(0, T ; L^{\infty}(\Omega)\right)} \leq c\left(M+L T^{\frac{1}{p}}\right)\left(1+T^{\frac{1}{p^{\prime}}}\left(M+L T^{\frac{1}{p}}\right)\right) .
$$

From now on we restrict ourselves to $T \leq 1$ and fix $R>c\left(1+L^{2}\right)$, where $c$ is the maximum of constants from (22), (26) and (27). Moreover, we assume that $M$ fulfills $R \geq c(1+L+M)^{2}$ and define the quantity $K$ as follows

$$
K=\sup _{s \in\left[0, R^{2}\right]} \sum_{j=0}^{3}\left|\mu^{(j)}(s)\right|+\sup _{t \in[-R, R]} \sum_{j=0}^{2}\left|\lambda^{(j)}(t)\right| .
$$

Obviously, the assumed smoothness of functions $\mu$ and $\lambda$ ensures that $K$ is finite. Furthermore, we define

$$
\Pi=\sup _{r \in[-\tilde{c} M, \tilde{c} M]} \sum_{j=0}^{2}\left\|\pi^{(j)}\left(\rho_{*}+\theta_{0}+r\right)\right\|_{L^{\infty}\left(Q_{T}\right)}
$$

where $\tilde{c}$ is such that $\|\theta\|_{L^{\infty}\left(0, T ; L^{\infty}(\Omega)\right)} \leq \tilde{c} M$ for all $(\theta, w) \in H_{T, M}$ (see also (28)). The fact that $\Pi$ is finite follows as $\rho_{*}+\theta_{0}$ is a continuous function by the Sobolev embedding and the assumption $\rho_{*}+\theta_{0} \in W^{1, q}(\Omega), q>d$. 
First, we check that $\Phi: H_{T, M} \rightarrow H_{T, M}$ if $T$ and $M$ are chosen appropriately. To this end we fix $(\vartheta, w) \in H_{T, M}$ and estimate $\mathcal{G}(\vartheta, w), \mathcal{F}(\vartheta, w)$ respectively. As $\vartheta(0)=0$, we get for $t \in[0, T]$ using the Hölder inequality

$$
\|\vartheta(t)\|_{W^{1, q}(\Omega)} \leq \int_{0}^{t}\left\|\partial_{t} \vartheta\right\|_{W^{1, q}(\Omega)} \mathrm{d} s \leq T^{\frac{1}{p^{f}}}\left\|\partial_{t} \vartheta\right\|_{L^{p}\left(0, T ; W^{1, q}(\Omega)\right)}
$$

and consequently

$$
\|\vartheta\|_{L^{\infty}\left(0, T ; W^{1, q}(\Omega)\right)} \leq T^{\frac{1}{p^{\prime}}\|\vartheta\|_{W^{1, p}\left(0, T ; W^{1, q}(\Omega)\right)}} .
$$

Let us focus on the estimate of $\mathcal{G}(\vartheta, w)$. Using the Sobolev embedding, (23), (28) and the Hölder inequality it follows that

$$
\begin{aligned}
\left\|\nabla w \cdot E_{w}\left(\rho_{*}+\theta_{0}+\vartheta\right)\right\|_{W^{1, q}(\Omega)} & \leq c\|w\|_{W^{2, q}(\Omega)}\left\|E_{w}\right\|_{W^{1, q}}\left\|\rho_{*}+\theta_{0}+\vartheta\right\|_{W^{1, q}(\Omega)} \\
& \leq c\|w\|_{W^{2, q}(\Omega)} T^{\frac{1}{p^{\prime}}}\|w\|_{L^{p}\left(0, T ; W^{2, q}(\Omega)\right)}\left(\left\|\rho_{*}+\theta_{0}\right\|_{W^{1, q}(\Omega)}+\|\vartheta\|_{W^{1, q}(\Omega)}\right) \\
& \leq c(M+L)^{2} T^{\frac{1}{p^{\prime}}}\|w\|_{W^{2, q}(\Omega)}
\end{aligned}
$$

and

$$
\|\vartheta \operatorname{div} w\|_{W^{1, q}(\Omega)} \leq c\|\vartheta\|_{W^{1, q}(\Omega)}\|w\|_{W^{2, q}(\Omega)} \leq c T^{\frac{1}{p^{\gamma}}} M\|w\|_{W^{2, q}(\Omega)} .
$$

In order to proceed with the bound of $\mathcal{F}(\vartheta, w)$ we begin with some preparatory work. The assumed smoothness of $\mu$ and $\lambda$ yields by the mean value theorem

$$
\begin{aligned}
\mu^{(k)}\left(|A|^{2}\right)-\mu^{(k)}\left(|B|^{2}\right) & =\int_{0}^{1} \frac{\mathrm{d}}{\mathrm{d} s} \mu^{(k)}\left(|s A+(1-s) B|^{2}\right) \mathrm{d} s \\
& =2 \int_{0}^{1} \mu^{(k+1)}\left(|s A+(1-s) B|^{2}\right)(s A+(1-s) B) \cdot(A-B) \mathrm{d} s k=0,1,2,
\end{aligned}
$$

$$
\begin{aligned}
& \mu^{\prime}\left(|A|^{2}\right)|A|^{2}-\mu^{\prime}\left(|B|^{2}\right)|B|^{2}=\int_{0}^{1} \frac{\mathrm{d}}{\mathrm{d} s}\left(\mu^{\prime}\left(|s A+(1-s) B|^{2}\right)|s A+(1-s) B|^{2}\right) \mathrm{d} s \\
& =2 \int_{0}^{1}(s A+(1-s) B) \cdot(A-B)\left(\mu^{\prime \prime}\left(|s A+(1-s) B|^{2}\right)|s A+(1-s) B|^{2}+\mu^{\prime}\left(|s A+(1-s) B|^{2}\right)\right) \mathrm{d} s
\end{aligned}
$$

$$
\begin{aligned}
\mu\left(|A|^{2}\right) \operatorname{div} A-\mu\left(|B|^{2}\right) \operatorname{div} B= & \int_{0}^{1} \frac{\mathrm{d}}{\mathrm{d} s}\left(\mu\left(|s A+(1-s) B|^{2}\right) \operatorname{div}(s A+(1-s) B)\right) \mathrm{d} s \\
= & \int_{0}^{1} 2 \mu^{\prime}\left(|s A+(1-s) B|^{2}\right)(s A+(1-s) B) \cdot(A-B) \operatorname{div}(s A+(1-s) B) \\
& +\mu\left(|s A+(1-s) B|^{2}\right) \operatorname{div}(A-B) \mathrm{d} s
\end{aligned}
$$


and

$$
\begin{aligned}
\mu^{\prime}\left(|A|^{2}\right)|A|^{2} \operatorname{div} A-\mu^{\prime}\left(|B|^{2}\right)|B|^{2} \operatorname{div} B \\
=\int_{0}^{1} \frac{\mathrm{d}}{\mathrm{d} s}\left(\mu^{\prime}\left(|s A+(1-s) B|^{2}\right)|s A+(1-s) B|^{2} \operatorname{div}(s A+(1-s) B)\right) \mathrm{d} s \\
=\int_{0}^{1} 2(s A+(1-s) B) \cdot(A-B)\left(\mu^{\prime \prime}\left(|s A+(1-s) B|^{2}\right)|s A+(1-s)|^{2} \operatorname{div}(s A+(1-s) B)\right. \\
\left.\quad+\mu^{\prime}\left(|s A+(1-s) B|^{2}\right)\right) \operatorname{div}(s A+(1-s) B) \\
\quad+\mu^{\prime}\left(|s A+(1-s) B|^{2}\right)|s A+(1-s) B|^{2} \operatorname{div}(A-B) \mathrm{d} s
\end{aligned}
$$

assuming $A, B$ are $d \times d$-matrix valued sufficiently smooth functions. Moreover, it follows that

$$
\begin{aligned}
& \lambda(a)-\lambda(b)=\int_{0}^{1} \lambda^{\prime}(s a+(1-s) b)(a-b) \mathrm{d} s, \\
& \lambda^{\prime}(a) a-\lambda^{\prime}(b) b=\int_{0}^{1} \frac{\mathrm{d}}{\mathrm{d} s}\left(\lambda^{\prime}(s a+(1-s) b)(s a+(1-s) b)\right) \mathrm{d} s \\
& =\int_{0}^{1}\left(\lambda^{\prime}(s a+(1-s) b)+\lambda^{\prime \prime}(s a+(1-s) b)(s a+(1-s) b)\right)(a-b) \mathrm{d} s, \\
& \lambda(a) \nabla a-\lambda(b) \nabla b=\int_{0}^{1} \frac{\mathrm{d}}{\mathrm{d} s}(\lambda(s a+(1-s) b) \nabla(s a+(1-s) b)) \mathrm{d} s \\
& =\int_{0}^{1} \lambda^{\prime}(s a+(1-s) b)(a-b) \nabla(s a+(1-s) b)+\lambda(s a+(1-s) b) \nabla(a-b) \mathrm{d} s \\
& \lambda^{\prime}(a) a \nabla a-\lambda^{\prime}(b) b \nabla b=\int_{0}^{1} \frac{\mathrm{d}}{\mathrm{d} s}\left(\lambda^{\prime}(s a+(1-s) b)(s a+(1-s) b) \nabla(s a+(1-s) b)\right) \mathrm{d} s \\
& =\int_{0}^{1} \lambda^{\prime \prime}(s a+(1-s) b)(a-b)(s a+(1-s) b) \nabla(s a+(1-s) b) \\
& +\lambda^{\prime}(s a+(1-s) b)(a-b) \nabla(s a+(1-s) b) \\
& +\lambda^{\prime}(s a+(1-s) b)(s a+(1-s) b) \nabla(a-b) \mathrm{d} s
\end{aligned}
$$

for arbitrary real valued functions $a, b$.

Recalling (44) we get using (31), (32), (35), (36), (37), (21) and the Jensen inequality $\left\|a_{j k}^{l m}(\mathbb{D} u)-a_{j k}^{l m}(\mathbb{D} v)\right\|_{L^{\infty}\left(0, T ; L^{\infty}(\Omega)\right)}$

$\leq K \int_{0}^{1}\left(1+\left\|s \mathbb{D}^{D} u+(1-s) \mathbb{D}^{D} v\right\|_{L^{\infty}\left(0, T ; L^{\infty}(\Omega)\right)}^{2}\right)\left\|s \mathbb{D}^{D} u+(1-s) \mathbb{D}^{D} v\right\|_{L^{\infty}\left(0, T ; L^{\infty}(\Omega)\right)}$

$\times\left\|\mathbb{D}^{D}(u-v)\right\|_{L^{\infty}\left(0, T ; L^{\infty}(\Omega)\right)}+\left(1+\|s \operatorname{div} u+(1-s) \operatorname{div} v\|_{L^{\infty}\left(0, T ; L^{\infty}(\Omega)\right)}\right)\|\operatorname{div}(u-v)\|_{L^{\infty}\left(0, T ; L^{\infty}(\Omega)\right)} \mathrm{d} s$ $\leq c K\left(1+\|u\|_{\mathcal{V}^{p, q}\left(Q_{T}\right)}+\|v\|_{\mathcal{V}^{p, q}\left(Q_{T}\right)}+\|u\|_{\mathcal{V}^{p, q}\left(Q_{T}\right)}^{2}+\|v\|_{\mathcal{V}^{p, q}\left(Q_{T}\right)}^{2}\right)\|u-v\|_{\mathcal{V}^{p, q}\left(Q_{T}\right)}$,

whenever $u, v \in \mathcal{V}^{p, q}\left(Q_{T}\right)$.

We can proceed with the bound of $(20)_{2}$. First, we estimate using (26), (27) and (24)

$$
\begin{aligned}
\left\|\operatorname{tr}\left(\tilde{\mathcal{S}}(w) E_{w}\right)\right\|_{L^{p}\left(0, T ; L^{q}(\Omega)\right)} & \leq K\left(\|\mathcal{G}(w)\|_{L^{\infty}\left(0, T ; L^{\infty}(\Omega)\right)}+\|\mathcal{D}(w)\|_{L^{\infty}\left(0, T ; L^{\infty}(\Omega)\right)}\right)\left\|E_{w}\right\|_{L^{p}\left(0, T ; L^{q}(\Omega)\right)} \\
& \leq c K T(M+L)^{2}(1+(M+L)) .
\end{aligned}
$$


Then we continue by expanding the divergence to arrive at

$$
\begin{aligned}
& \operatorname{div}\left(\tilde{\mathcal{S}}(w)-\mathcal{S}\left(\mathbb{D}^{D} w\right)\right)=\mu\left(|\mathcal{G}(w)|^{2}\right) \operatorname{div} \mathcal{G}(w)-\mu\left(\left|\mathbb{D}^{D} w\right|^{2}\right) \operatorname{div} \mathbb{D}^{D} w \\
& +2 \mu^{\prime}\left(|\mathcal{G}(w)|^{2}\right)|\mathcal{G}(w)|^{2} \operatorname{div} \mathcal{G}(w)-2 \mu\left(\left|\mathbb{D}^{D} w\right|^{2}\right)\left|\mathbb{D}^{D} w\right|^{2} \operatorname{div} \mathbb{D}^{D} w \\
& +\lambda(\mathcal{D}(w)) \nabla \mathcal{D}(w)-\lambda(\operatorname{div} w) \nabla \operatorname{div} w \\
& +\lambda^{\prime}(\mathcal{D}(w)) \mathcal{D}(w) \nabla \mathcal{D}(w)-\lambda^{\prime}(\operatorname{div} w) \operatorname{div} w \nabla \operatorname{div} w=\sum_{i=1}^{4} I_{j} .
\end{aligned}
$$

We estimate each $I_{j}$ separately. We observe that denoting

$$
\mathcal{G}_{s}=s\left(\mathbb{D}^{D} w-\operatorname{sym}\left(\nabla w E_{w}\right)^{D}\right)+(1-s) \mathbb{D}^{D} w
$$

it follows by (33) that

$$
I_{1}=-\int_{0}^{1} 2 \mu^{\prime}\left(\left|\mathcal{G}_{s}\right|^{2}\right) \mathcal{G}_{s} \cdot \operatorname{sym}\left(\nabla w E_{w}\right)^{D} \operatorname{div} \mathcal{G}_{s}+\mu\left(\left|\mathcal{G}_{s}\right|^{2}\right) \operatorname{div} \operatorname{sym}\left(\nabla w E_{w}\right)^{D} \mathrm{~d} s .
$$

Further we employ the estimates

$$
\begin{aligned}
\left\|\mathcal{G}_{s}\right\|_{L^{\infty}\left(0, T ; L^{\infty}(\Omega)\right)} & \leq c\left\|\mathbb{D}^{D} w\right\|_{L^{\infty}\left(0, T ; L^{\infty}(\Omega)\right)}+\|\nabla w\|_{L^{\infty}\left(0, T ; L^{\infty}(\Omega)\right)}\left\|E_{w}\right\|_{L^{\infty}\left(0, T ; L^{\infty}(\Omega)\right)} \\
& \leq c\|w\|_{\mathcal{V}^{p, q}\left(Q_{T}\right)}\left(1+T^{\frac{1}{p^{\prime}}}\|w\|_{\mathcal{V}^{p, q}\left(Q_{T}\right)}\right)
\end{aligned}
$$

$\left\|\operatorname{div} \mathcal{G}_{s}\right\|_{L^{p}\left(0, T ; L^{q}(\Omega)\right)} \leq c\|w\|_{L^{p}\left(0, T ; L^{q}(\Omega)\right)}\left(1+\left\|E_{w}\right\|_{L^{\infty}\left(0, T ; L^{\infty}(\Omega)\right)}\right)+\|\nabla w\|_{L^{\infty}\left(0, T ; L^{\infty}(\Omega)\right)}\left\|\nabla E_{w}\right\|_{L^{p}\left(0, T ; L^{q}(\Omega)\right)}$

$$
\leq c\|w\|_{\mathcal{V}^{p, q}\left(Q_{T}\right)}\left(1+\left(T^{\frac{1}{p^{\prime}}}+T\right)\|w\|_{\mathcal{V}^{p, q}\left(Q_{T}\right)}\right),
$$

$$
\begin{aligned}
\left\|\operatorname{div} \operatorname{sym}\left(\nabla w E_{w}\right)^{D}\right\|_{L^{p}\left(0, T ; L^{q}(\Omega)\right)} \leq & c\left\|\nabla^{2} w\right\|_{L^{p}\left(0, T ; L^{q}(\Omega)\right)}\left\|E_{w}\right\|_{L^{\infty}\left(0, T ; L^{\infty}(\Omega)\right)} \\
& +\|\nabla w\|_{L^{\infty}\left(0, T ; L^{\infty}(\Omega)\right)}\left\|\nabla E_{w}\right\|_{L^{p}\left(0, T ; L^{q}(\Omega)\right)} \\
\leq & \|w\|_{\mathcal{V}^{p, q}\left(Q_{T}\right)}^{2}\left(T^{\frac{1}{p^{\prime}}}+T\right)
\end{aligned}
$$

that follow by (23), (25) and (21). Employing the Jensen inequality, (40), (41) and (42) we infer

$$
\begin{aligned}
& \quad\left\|I_{1}\right\|_{L^{p}\left(0, T ; L^{q}(\Omega)\right)} \\
& \leq c K\|w\|_{\mathcal{V}^{p, q}\left(Q_{T}\right)}^{2}\left(1+T^{\frac{1}{p^{\prime}}}\|w\|_{\mathcal{V}^{p, q}\left(Q_{T}\right)}\right)\|\nabla w\|_{L^{\infty}\left(0, T ; L^{\infty}(\Omega)\right)}\left\|E_{w}\right\|_{L^{\infty}\left(0, T ; L^{\infty}(\Omega)\right)}\left(1+\left(T^{\frac{1}{p^{\prime}}}+T\right)\|w\|_{\mathcal{V}^{p, q}\left(Q_{T}\right)}\right) \\
& \quad+c K\|w\|_{\mathcal{V}^{p, q}\left(Q_{T}\right)}^{2}\left(T^{\frac{1}{p^{\prime}}}+T\right) \\
& \leq c K\|w\|_{\mathcal{V}^{p, q}\left(Q_{T}\right)}^{2}\left(\left(1+T^{\frac{1}{p^{\prime}}}\|w\|_{\mathcal{V}^{p, q}\left(Q_{T}\right)}\right) T^{\frac{1}{p^{\prime}}}\|w\|_{\mathcal{V}^{p, q}\left(Q_{T}\right)}^{2}\left(1+\left(T^{\frac{1}{p^{\prime}}}+T\right)\|w\|_{\mathcal{V}^{p, q}\left(Q_{T}\right)}\right)+T^{\frac{1}{p^{\prime}}}+T\right) \\
& \leq c K(M+L)^{2}\left((1+(M+L))^{2} T^{\frac{1}{p^{\prime}}}(M+L)^{2}+T^{\frac{1}{p^{\prime}}}+T\right)
\end{aligned}
$$

where the Sobolev embedding, interpolation inequality (21) and (25) were also employed. By (34) we have

$$
\begin{aligned}
I_{2}=-\int_{0}^{1} 2\left(\mu^{\prime \prime}\left(\left|\mathcal{G}_{s}\right|^{2}\right)\left|\mathcal{G}_{s}\right|^{2}+\mu^{\prime}\left(\left|\mathcal{G}_{s}\right|^{2}\right)\right) \mathcal{G}_{s} \cdot \operatorname{sym}\left(\nabla w E_{w}\right)^{D} & \operatorname{div} \mathcal{G}_{s} \\
& +\mu\left(\left|\mathcal{G}_{s}\right|^{2}\right)\left|\mathcal{G}_{s}\right|^{2} \operatorname{div} \operatorname{sym}\left(\nabla w E_{w}\right)^{D} \mathrm{~d} s .
\end{aligned}
$$


Then using (40), (41) and (42) we obtain

$$
\begin{aligned}
& \quad\left\|I_{2}\right\|_{L^{p}\left(0, T ; L^{q}(\Omega)\right)} \\
& \leq c K\left(\left(\|w\|_{\mathcal{V}^{p, q}\left(Q_{T}\right)}^{2}\left(1+T^{\frac{1}{p^{\prime}}}\|w\|_{\mathcal{V}^{p, q}\left(Q_{T}\right)}\right)^{2}+1\right)\|w\|_{\mathcal{V}^{p, q}\left(Q_{T}\right)}\left(1+T^{\frac{1}{p^{\prime}}}\|w\|_{\mathcal{V}^{p, q}\left(Q_{T}\right)}\right)\|\nabla w\|_{L^{\infty}\left(0, T ; L^{\infty}(\Omega)\right)}\right. \\
& \left.\quad \times\left\|E_{w}\right\|_{L^{\infty}\left(0, T ; L^{\infty}(\Omega)\right)}\|w\|_{\mathcal{V}^{p, q}\left(Q_{T}\right)}\left(1+\left(T^{\frac{1}{p^{\prime}}}+T\right)\|w\|_{\mathcal{V}^{p, q}\left(Q_{T}\right)}\right)+\|w\|_{\mathcal{V}^{p, q}\left(Q_{T}\right)}^{4}\left(1+T^{\frac{1}{p^{\prime}}}\|w\|_{\mathcal{V}^{p, q}\left(Q_{T}\right)}\right)\left(T^{\frac{1}{p^{\prime}}}+T\right)\right) \\
& \leq c K\left(\left((M+L)^{2}\left(1+(M+L)^{2}\right)+1\right)(M+L)^{4}(1+(M+L)) T^{\frac{1}{p^{\prime}}}(1+(M+L))\right. \\
& \left.\quad+(M+L)^{4}(1+(M+L))\left(T^{\frac{1}{p^{\prime}}}+T\right)\right) .
\end{aligned}
$$

Denoting

$$
\mathcal{D}_{s}=s\left(\operatorname{div} w-\nabla w \cdot E_{w}\right)+(1-s) \operatorname{div} w
$$

we obtain by (36)

$$
I_{3}=-\int_{0}^{1} \lambda^{\prime}\left(\mathcal{D}_{s}\right) \nabla w \cdot E_{w} \nabla \mathcal{D}_{s}+\lambda\left(\mathcal{D}_{s}\right) \nabla\left(\nabla w \cdot E_{w}\right) .
$$

Moreover, one has by (21) and (23)

$$
\begin{aligned}
\left\|\mathcal{D}_{s}\right\|_{L^{\infty}\left(0, T ; L^{\infty}(\Omega)\right)} & \leq c\|\nabla w\|_{L^{\infty}\left(0, T ; L^{\infty}(\Omega)\right)}\left(1+\left\|E_{w}\right\|_{L^{\infty}\left(0, T ; L^{\infty}(\Omega)\right)}\right) \\
& \leq c\|w\|_{\mathcal{V}^{p, q}\left(Q_{T}\right)}\left(1+T^{\frac{1}{p^{\prime}}}\|w\|_{\mathcal{V}^{p, q}\left(Q_{T}\right)}\right)
\end{aligned}
$$

and

$$
\begin{aligned}
\left\|\nabla \mathcal{D}_{s}\right\|_{L^{p}\left(0, T ; L^{q}(\Omega)\right)} \leq & c\left\|\nabla^{2} w\right\|_{L^{p}\left(0, T ; W^{2, q}(\Omega)\right)}+\left\|\nabla^{2} w\right\|_{L^{p}\left(0, T ; W^{2, q}(\Omega)\right)}\left\|E_{w}\right\|_{L^{\infty}\left(0, T ; L^{\infty}(\Omega)\right)} \\
& +\|\nabla w\|_{L^{\infty}\left(0, T ; L^{\infty}(\Omega)\right)}\left\|\nabla E_{w}\right\|_{L^{p}\left(0, T ; L^{q}(\Omega)\right)} \\
\leq & \|w\|_{\mathcal{V}^{p, q}\left(Q_{T}\right)}\left(1+\left(T^{\frac{1}{p^{\prime}}}+T\right)\|w\|_{\mathcal{V}^{p, q}\left(Q_{T}\right)}\right) .
\end{aligned}
$$

Then it follows by (21), (23), (45) and (46)

$$
\begin{aligned}
\left\|I_{3}\right\|_{L^{p}\left(0, T ; L^{q}(\Omega)\right) \leq} \leq & c K\left(\|\nabla w\|_{L^{\infty}\left(0, T ; L^{\infty}(\Omega)\right)}\left\|E_{w}\right\|_{L^{\infty}\left(0, T ; L^{\infty}(\Omega)\right)}\left\|\nabla \mathcal{D}_{s}\right\|_{L^{p}\left(0, T ; L^{q}(\Omega)\right)}\right. \\
& \left.+\left\|\nabla^{2} w\right\|_{L^{p}\left(0, T ; L^{q}(\Omega)\right)}\left\|E_{w}\right\|_{L^{\infty}\left(0, T ; L^{\infty}(\Omega)\right)}+\|\nabla w\|_{L^{\infty}\left(0, T ; L^{\infty}(\Omega)\right)}\left\|\nabla E_{w}\right\|_{L^{p}\left(0, T ; L^{q}(\Omega)\right)}\right) \\
\leq & c K\left(T^{\frac{1}{p^{\prime}}}\|w\|_{\mathcal{V}^{p, q}\left(Q_{T}\right)}^{3}\left(1+\left(T^{\frac{1}{p^{\prime}}}+T\right)\|w\|_{\mathcal{V}^{p, q}\left(Q_{T}\right)}\right)+\left(T^{\frac{1}{p^{\prime}}}+T\right)\|w\|_{\mathcal{V}^{p, q}\left(Q_{T}\right)}^{2}\right) \\
\leq & c K\left(T^{\frac{1}{p^{\prime}}}(M+L)^{3}(1+(M+L))+\left(T^{\frac{1}{p}}+T\right)(M+L)^{2}\right) .
\end{aligned}
$$

By (37) we obtain

$$
\begin{aligned}
I_{4}= & -\int_{0}^{1} \lambda^{\prime \prime}\left(\mathcal{D}_{s}\right) \nabla w \cdot E_{w} \mathcal{D}_{s} \nabla \mathcal{D}_{s}+\lambda^{\prime}\left(\mathcal{D}_{s}\right) \nabla w \cdot E_{w} \nabla \mathcal{D}_{s} \\
& +\lambda^{\prime}\left(\mathcal{D}_{s}\right) \mathcal{D}_{s} \nabla\left(\nabla w \cdot E_{w}\right) \mathrm{d} s .
\end{aligned}
$$


Finally, we get by (27) and (21)

$$
\begin{aligned}
& \left\|I_{4}\right\|_{L^{p}\left(0, T ; L^{q}(\Omega)\right)} \\
& \leq c K\left(\|\nabla w\|_{L^{\infty}\left(0, T ; L^{\infty}(\Omega)\right)}\left\|E_{w}\right\|_{L^{\infty}\left(0, T ; L^{\infty}(\Omega)\right)}\left(1+\left\|\mathcal{D}_{s}\right\|_{L^{\infty}\left(0, T ; L^{\infty}(\Omega)\right)}\right)\left\|\nabla \mathcal{D}_{s}\right\|_{L^{p}\left(0, T ; L^{q}(\Omega)\right)}\right. \\
& +\left\|\mathcal{D}_{s}\right\|_{L^{\infty}\left(0, T ; L^{\infty}(\Omega)\right)}\left(\left\|\nabla^{2} w\right\|_{L^{p}\left(0, T ; L^{q}(\Omega)\right)}\left\|E_{w}\right\|_{L^{\infty}\left(0, T ; L^{\infty}(\Omega)\right)}+\|\nabla w\|_{L^{\infty}\left(0, T ; L^{\infty}(\Omega)\right)}\left\|\nabla E_{w}\right\|_{L^{p}\left(0, T ; L^{q}(\Omega)\right)}\right) \\
& \leq c K\left(T^{\frac{1}{p^{\prime}}}\|w\|_{\mathcal{V}^{p, q}\left(Q_{T}\right)}^{4}\left(1+T^{\frac{1}{p^{\prime}}}\|w\|_{\mathcal{V}^{p, q}\left(Q_{T}\right)}\right)\left(1+\left(T^{\frac{1}{p^{\prime}}}+T\right)\|w\|_{\mathcal{V}^{p, q}\left(Q_{T}\right)}\right)\right. \\
& \left.+\left(T^{\frac{1}{p^{\prime}}}+T\right)\|w\|_{\mathcal{V}^{p, q}\left(Q_{T}\right)}^{3}\left(1+T^{\frac{1}{p^{\prime}}}\|w\|_{\mathcal{V}^{p, q}\left(Q_{T}\right)}\right)\right) \\
& \leq c K\left(T^{\frac{1}{p^{\prime}}}(M+L)^{4}(1+(M+L))^{2}+\left(T^{\frac{1}{p^{\prime}}}+T\right)(1+M+L)^{3}\right) .
\end{aligned}
$$

In order to proceed with the estimate of $\mathcal{F}$ we have

$$
\begin{aligned}
& \left\|\pi^{\prime}\left(\rho_{*}+\theta_{0}+\vartheta\right) \nabla\left(\theta_{0}+\vartheta\right) E_{w}\right\|_{L^{p}\left(0, T ; L^{q}(\Omega)\right)} \leq \Pi\left\|\nabla\left(\theta_{0}+\vartheta\right)\right\|_{L^{p}\left(0, T ; L^{q}(\Omega)\right)}\left\|E_{w}\right\|_{L^{\infty}\left(0, T ; L^{\infty}(\Omega)\right)} \\
& \leq c \Pi(L+M) T^{\frac{1}{p^{\prime}}}(M+L) .
\end{aligned}
$$

Applying (28) and the Sobolev embedding we obtain

$$
\left\|\vartheta \partial_{t} w\right\|_{L^{p}\left(0, T ; L^{q}(\Omega)\right)} \leq\|\vartheta\|_{L^{\infty}\left(0, T ; L^{\infty}(\Omega)\right)}\left\|\partial_{t} w\right\|_{L^{p}\left(0, T ; L^{q}(\Omega)\right)} \leq c T^{\frac{1}{p^{\prime}}} M(M+L) .
$$

One immediately has

$$
\left\|\pi^{\prime}\left(\rho_{*}+\theta_{0}\right) \nabla \theta_{0}\right\|_{L^{p}\left(0, T ; L^{q}(\Omega)\right)} \leq c T^{\frac{1}{p}} \Pi L .
$$

By the assumed smoothness of $\pi$, the Sobolev embedding and (28) we get

$$
\begin{aligned}
\left\|\left(\pi^{\prime}\left(\rho_{*}+\theta_{0}+\vartheta\right)-\pi^{\prime}\left(\rho_{*}+\theta_{0}\right)\right) \nabla\left(\theta_{0}+\vartheta\right)\right\|_{L^{p}\left(0, T ; L^{q}(\Omega)\right)} & \leq \Pi\|\vartheta\|_{L^{\infty}\left(0, T ; L^{\infty}(\Omega)\right)}\left\|\nabla\left(\theta_{0}+\vartheta\right)\right\|_{L^{p}\left(0, T ; L^{q}(\Omega)\right)} \\
& \leq c \Pi T^{\frac{1}{p^{\prime}}} M(L+M)
\end{aligned}
$$

It remains to estimate the norm of the difference $\operatorname{div} \mathcal{S}\left(\mathbb{D}^{D} w\right)-\mathcal{A}\left(\mathbb{D} u_{0}\right)(\mathbb{D} w)$. To this end, recalling (3) and (66) we obtain

$$
\begin{aligned}
\left\|\operatorname{div} \mathcal{S}\left(\mathbb{D}^{D} w\right)-\mathcal{A}\left(\mathbb{D} u_{0}\right)\left(\mathbb{D}\left(w-u_{0}\right)\right)\right\|_{L^{p}\left(0, T ; L^{q}(\Omega)\right)} \leq & \sum_{j, k, l, m=1}^{d}\left(a_{j k}^{l m}(\mathbb{D} w)-a_{j k}^{l m}\left(\mathbb{D} u_{0}\right)\right) \partial_{l} \partial_{m}\left(w-u_{0}\right) \|_{L^{p}\left(0, T ; L^{q}(\Omega)\right)} \\
& +\left\|\sum_{j, k, l, m=1}^{d} a_{j k}^{l m}(\mathbb{D} w) \partial_{l} \partial_{m} u_{0}\right\|_{L^{p}\left(0, T ; L^{q}(\Omega)\right)}
\end{aligned}
$$

By (41) and (38) it follows that

$$
\begin{aligned}
& \left\|a_{j k}^{l m}(\mathbb{D} w)-a_{j k}^{l m}\left(\mathbb{D} u_{0}\right)\right\|_{L^{\infty}\left(0, T ; L^{\infty}(\Omega)\right)} \\
& \leq c K\left(1+\|w\|_{\mathcal{V}^{p, q}\left(Q_{T}\right)}+\left\|u_{0}\right\|_{W^{2,1}(\Omega)}+\|w\|_{\mathcal{V}^{p, q}\left(Q_{T}\right)}^{2}+\left\|u_{0}\right\|_{W^{2, q}(\Omega)}^{2}\right)\left\|w-u_{0}\right\|_{\mathcal{V}^{p, q}\left(Q_{T}\right)} \text {. }
\end{aligned}
$$

We use the above estimate and (21) in (53) to deduce

$$
\begin{aligned}
\| \operatorname{div} \mathcal{S}\left(\mathbb{D}^{D} w\right)- & \mathcal{A}\left(\mathbb{D} u_{0}\right)\left(\mathbb{D}\left(w-u_{0}\right)\right) \|_{L^{p}\left(0, T ; L^{q}(\Omega)\right)} \leq c K\left(1+L+L^{2}+M+M^{2}\right) M^{2} \\
& +c K\left(1+(M+L)+(M+L)^{2}\right) L T^{\frac{1}{p}}
\end{aligned}
$$


We summarize (29), (30), (39), (43), (44), (47), (48), (50), (49), (51), (52) and (54) to conclude

$$
\begin{aligned}
& \|\mathcal{G}(\vartheta, w)\|_{L^{p}\left(0, T ; W^{1, q}(\Omega)\right)}+\|\mathcal{F}(\vartheta, w)\|_{L^{p}\left(0, T ; L^{q}(\Omega)\right)} \\
& \quad \leq c\left(\sum_{j=1}^{N_{1}} K^{k_{j}} L^{l_{j}} M^{m_{j}} \Pi^{\left|k_{j}-1\right|} T^{\alpha_{j}}+\sum_{j=1}^{N_{2}} K^{n_{j}} L^{o_{j}} M^{p_{j}}+T\right),
\end{aligned}
$$

where $\alpha_{j}>0, l_{j}, m_{j}, o_{j}, \in \mathbf{N} \cup\{0\}, p_{j} \in \mathbf{N} \backslash\{1\}, k_{j}, n_{j} \in\{0,1\}$.

The $M$ appears on the right hand side either to some nonnegative power in a product with a nonnegative power of $T$ or to the second or higher power not multiplied by a nonnegative power of $T$. As a result, for every $c>0$ we can choose $M$ and consequently also $T$ in such way that

$$
\|\mathcal{G}(\vartheta, w)\|_{L^{p}\left(0, T ; W^{1, q}(\Omega)\right)}+\|\mathcal{F}(\vartheta, w)\|_{L^{p}\left(0, T ; L^{q}(\Omega)\right)} \leq c M .
$$

Theorem 3.1 then yields $\Phi: H_{T, M} \mapsto H_{T, M}$ for these appropriately chosen $T$ and $M$.

In order to verify that $\Phi$ is a contraction on $H_{T, M}$ we fix arbitrary pairs $\left(\vartheta^{1}, w^{1}\right),\left(\vartheta^{2}, w^{2}\right) \in$ $H_{T, M}$. Next we note that the difference $\left(\theta^{1}-\theta^{2}, \tilde{u}^{1}-\tilde{u}^{2}\right)$ of solutions corresponding to $\left(\vartheta^{1}, w^{1}\right)$, $\left(\vartheta^{2}, w^{2}\right)$ respectively, solves the equations

$$
\begin{aligned}
\partial_{t}\left(\theta^{1}-\theta^{2}\right)+\left(\rho_{*}+\theta_{0}\right) \operatorname{div}\left(\tilde{u}^{1}-\tilde{u}^{2}\right) & =\mathcal{G}\left(\vartheta^{1}, w^{1}\right)-\mathcal{G}\left(\vartheta^{2}, w^{2}\right), \\
\left(\rho_{*}+\theta_{0}\right) \partial_{t}\left(w^{1}-w^{2}\right)-\mathcal{A}\left(\mathbb{D} u_{0}\right)\left(\mathbb{D}\left(\tilde{u}^{1}-\tilde{u}^{2}\right)\right)+\pi^{\prime}\left(\rho_{0}+\theta_{0}\right) \nabla\left(\theta^{1}-\theta^{2}\right) & =\mathcal{F}\left(\vartheta^{1}, w^{1}\right)-\mathcal{F}\left(\vartheta^{2}, w^{2}\right) .
\end{aligned}
$$

We begin with the estimate of the difference on the right hand side of (55) $)_{1}$. Employing (28), the Sobolev embedding and (21) it follows that

$$
\begin{aligned}
\left\|\vartheta^{1} \operatorname{div} w^{1}-\vartheta^{2} \operatorname{div} w^{2}\right\|_{L^{p}\left(0, T ; W^{1, q}(\Omega)\right)} \leq & c\left\|\vartheta^{1}-\vartheta^{2}\right\|_{L^{\infty}\left(0, T ; L^{\infty}(\Omega)\right)}\left\|w^{1}\right\|_{L^{p}\left(0, T ; W^{2, q}(\Omega)\right)} \\
& +c\left\|\vartheta^{2}\right\|_{L^{\infty}\left(0, T ; L^{\infty}(\Omega)\right)}\left\|w^{1}-w^{2}\right\|_{L^{p}\left(0, T ; W^{2, q}(\Omega)\right)} \\
\leq & c T^{\frac{1}{p^{\prime}}}(M+L)\left\|\vartheta^{1}-\vartheta^{2}\right\|_{L^{\infty}\left(0, T ; L^{\infty}\right)} \\
& +c T^{\frac{1}{p^{\prime}}} M\left\|w^{1}-w^{2}\right\|_{L^{p}\left(0, T ; W^{2, q}(\Omega)\right)}
\end{aligned}
$$

as well as

$$
\begin{aligned}
& \left\|\nabla w^{1} \cdot E_{w^{1}}\left(\rho_{*}+\theta_{0}+\vartheta^{1}\right)-\nabla w^{2} \cdot E_{w^{2}}\left(\rho_{*}+\theta_{0}+\vartheta^{2}\right)\right\|_{L^{p}\left(0, T ; W^{1, q}(\Omega)\right)} \\
& \leq\left\|w^{1}-w^{2}\right\|_{L^{p}\left(0, T ; W^{2, q}\right)}\left\|E_{w^{1}}\right\|_{L^{\infty}\left(0, T ; L^{\infty}(\Omega)\right)}\left\|\rho_{*}+\theta_{0}+\vartheta^{1}\right\|_{L^{\infty}\left(0, T ; L^{\infty}(\Omega)\right)} \\
& +\left\|w^{2}\right\|_{L^{\infty}\left(0, T ; W^{1, \infty}(\Omega)\right.}\left\|E_{w^{1}}-E_{w^{2}}\right\|_{L^{p}\left(0, T ; W^{1, q}(\Omega)\right)}\left\|\rho_{*}+\theta_{0}+\vartheta^{1}\right\|_{L^{\infty}\left(0, T ; L^{\infty}(\Omega)\right)} \\
& +\left\|w^{2}\right\|_{L^{\infty}\left(0, T ; W^{1, \infty}(\Omega)\right.}\left\|E_{w^{2}}\right\|_{L^{\infty}\left(0, T ; L^{\infty}(\Omega)\right.}\left\|\vartheta^{1}-\vartheta^{2}\right\|_{L^{p}\left(0, T ; W^{1, q}(\Omega)\right)} \\
& \leq c M T^{\frac{1}{p^{\prime}}}\left\|w^{1}-w^{2}\right\|_{L^{p}\left(0, T ; W^{2, q}(\Omega)\right)}(L+M)+c(M+L)^{2} T\left\|w^{1}-w^{2}\right\|_{L^{p}\left(0, T ; W^{2, q}(\Omega)\right)} \\
& +c(M+L) M T^{\frac{1}{p^{\prime}}}\left\|\vartheta^{1}-\vartheta^{2}\right\|_{L^{p}\left(0, T ; W^{1, q}(\Omega)\right)}
\end{aligned}
$$

where (25) was also applied.

Next we focus on te estimate of the difference on the right hand side in $(55)_{2}$. By the assumed smoothness of $\pi$ we get

$$
\begin{aligned}
\| \nabla \pi\left(\rho_{*}+\theta_{0}+\vartheta^{1}\right) E_{w^{1}} & -\nabla \pi\left(\rho_{*}+\theta_{0}+\vartheta^{2}\right) E_{w^{2}} \|_{L^{p}\left(0, T ; L^{q}(\Omega)\right)} \\
& \leq \Pi\left\|\vartheta^{1}-\vartheta^{2}\right\|_{L^{p}\left(0, T ; L^{q}(\Omega)\right)}\left\|E_{w^{1}}\right\|_{L^{\infty}\left(0, T ; L^{\infty}(\Omega)\right)}+\Pi\left\|E_{w^{1}}-E_{w^{2}}\right\|_{L^{p}\left(0, T ; L^{q}(\Omega)\right)} \\
& \leq c \Pi\left(T^{\frac{1}{p^{\prime}}}(M+L)+T\right)\left[\vartheta^{1}-\vartheta^{2}, w^{1}-w^{2}\right]_{T, M} .
\end{aligned}
$$


In order to proceed, we observe that by (24) and (25)

$$
\begin{aligned}
& \left\|\mathcal{G}\left(w^{1}\right)-\mathcal{G}\left(w^{2}\right)\right\|_{L^{\infty}\left(0, T ; L^{\infty}(\Omega)\right)} \\
& \leq \leq\left\|\mathbb{D}^{D} w^{1}-\mathbb{D}^{D} w^{2}\right\|_{L^{\infty}\left(0, T ; L^{\infty}(\Omega)\right)}+\left\|\operatorname{sym}\left(\nabla\left(w^{1}-w^{2}\right) E_{w^{1}}\right)^{D}\right\|_{L^{\infty}\left(0, T ; L^{\infty}(\Omega)\right)} \\
& \quad+\left\|\operatorname{sym}\left(\nabla w^{2} E_{w^{1}-w^{2}}\right)^{D}\right\|_{L^{\infty}\left(0, T ; L^{\infty}(\Omega)\right)} \\
& \leq c\left\|w^{1}-w^{2}\right\|_{\mathcal{V}^{p, q}\left(Q_{T}\right)}\left(1+T^{\frac{1}{p^{\prime}}}\left\|w^{1}\right\|_{L^{p}\left(0, T ; W^{2, q}(\Omega)\right)}+T\left\|w^{2}\right\|_{L^{\infty}\left(0, T ; W^{1, \infty}(\Omega) f\right)}\right) .
\end{aligned}
$$

Similarly, we deduce

$$
\begin{aligned}
& \left\|\mathcal{D}\left(w^{1}\right)-\mathcal{D}\left(w^{2}\right)\right\|_{L^{\infty}\left(0, T ; L^{\infty}(\Omega)\right)} \\
& \quad \leq\left\|\operatorname{div}\left(w^{1}-w^{2}\right)\right\|_{L^{\infty}\left(0, T ; L^{\infty}(\Omega)\right)}+\left\|\nabla\left(w^{1}-w^{2}\right) \cdot E_{w^{1}}\right\|_{L^{\infty}\left(0, T ; L^{\infty}(\Omega)\right)} \\
& \quad+\left\|\nabla w^{2} \cdot E_{w^{1}-w^{2}}\right\|_{L^{\infty}\left(0, T ; L^{\infty}(\Omega)\right)} \\
& \leq c\left\|w^{1}-w^{2}\right\|_{\mathcal{V}^{p, q}\left(Q_{T}\right)}\left(1+T^{\frac{1}{p^{p}}}\left\|w^{1}\right\|_{L^{p}\left(0, T ; W^{2, q}(\Omega)\right)}+T\left\|w^{2}\right\|_{L^{\infty}\left(0, T ; W^{1, \infty}(\Omega)\right)}\right) .
\end{aligned}
$$

We employ the latter two estimates, (26), (27), (31) and (21) to obtain

$$
\begin{aligned}
\| & \operatorname{tr} \tilde{\mathcal{S}}\left(w^{1}\right) E_{w^{1}}-\operatorname{tr} \tilde{\mathcal{S}}\left(w^{2}\right) E_{w^{2}} \|_{L^{p}\left(0, T ; L^{q}(\Omega)\right)} \\
\leq & \left(\left\|\mu\left(\left|\mathcal{G}\left(w^{1}\right)\right|^{2}\right)\right\|_{L^{\infty}\left(0, T ; L^{\infty}(\Omega)\right)}\left\|\mathcal{G}\left(w^{1}\right)-\mathcal{G}\left(w^{2}\right)\right\|_{L^{\infty}\left(0, T ; L^{\infty}(\Omega)\right)}\right. \\
& +\| \lambda\left(\mathcal{D}\left(w^{1}\right)\left\|_{L^{\infty}\left(0, T ; L^{\infty}(\Omega)\right)}\right\| \mathcal{D}\left(w^{1}\right)-\mathcal{D}\left(w^{2}\right) \|_{L^{\infty}\left(0, T ; L^{\infty}(\Omega)\right)}\right. \\
& +\left\|\mu\left(\left|\mathcal{G}\left(w^{1}\right)\right|^{2}\right)-\mu\left(\left|\mathcal{G}\left(w^{2}\right)\right|^{2}\right)\right\|_{L^{\infty}\left(0, T ; L^{\infty}(\Omega)\right)}\left\|\mathcal{G}\left(w^{2}\right)\right\|_{L^{\infty}\left(0, T ; L^{\infty}(\Omega)\right)} \\
& \left.+\left\|\lambda\left(\mathcal{D}\left(w^{1}\right)\right)-\lambda\left(\mathcal{D}\left(w^{2}\right)\right)\right\|_{L^{\infty}\left(0, T ; L^{\infty}(\Omega)\right)}\left\|\mathcal{D}\left(w^{2}\right)\right\|_{L^{\infty}\left(0, T ; L^{\infty}(\Omega)\right)}\right)\left\|E_{w^{1}}\right\|_{L^{p}\left(0, T ; L^{q}(\Omega)\right)} \\
& +\left(\left\|\mu\left(\left|\mathcal{G}\left(w^{2}\right)\right|^{2}\right)\right\|_{L^{\infty}\left(0, T ; L^{\infty}(\Omega)\right)}\left\|\mathcal{G}\left(w^{2}\right)\right\|_{L^{\infty}\left(0, T ; L^{\infty}(\Omega)\right)}\right. \\
& \left.+\left\|\lambda\left(\mathcal{D}\left(w^{2}\right)\right)\right\|_{L^{\infty}\left(0, T ; L^{\infty}(\Omega)\right)}\left\|\mathcal{D}\left(w^{2}\right)\right\|_{L^{\infty}\left(0, T ; L^{\infty}(\Omega)\right)}\right)\left\|E_{w^{1}-w^{2}}\right\|_{L^{p}\left(0, T ; L^{q}(\Omega)\right)} \\
\leq & c K\left(1+(M+L)+(M+L)^{2}(1+(M+L))^{2}\right. \\
& +(M+L)(1+(M+L))) T(M+L)\left\|w^{1}-w^{2}\right\|_{\mathcal{V}^{p, q}\left(Q_{T}\right)} .
\end{aligned}
$$

We continue with expanding the divergence to obtain

$$
\begin{aligned}
\operatorname{div} & \left(\tilde{\mathcal{S}}\left(w^{1}\right)-\tilde{\mathcal{S}}\left(w^{2}\right)-\left(\mathcal{S}\left(\mathbb{D}^{D} w^{1}\right)-\mathcal{S}\left(\mathbb{D}^{D} w^{2}\right)\right)\right) \\
= & \left(\mu\left(\left|\mathcal{G}\left(w^{1}\right)\right|^{2}\right) \operatorname{div} \mathcal{G}\left(w^{1}\right)-\mu\left(\left|\mathbb{D}^{D} w^{1}\right|^{2}\right) \operatorname{div} \mathbb{D}^{D} w^{1}\right. \\
& \left.-\left(\mu\left(\left|\mathcal{G}\left(w^{2}\right)\right|^{2}\right) \operatorname{div} \mathcal{G}\left(w^{2}\right)-\mu\left(\left|\mathbb{D}^{D} w^{2}\right|^{2}\right) \operatorname{div} \mathbb{D}^{D} w^{2}\right)\right) \\
& +2\left(\mu^{\prime}\left(\left|\mathcal{G}\left(w^{1}\right)\right|^{2}\right)\left|\mathcal{G}\left(w^{1}\right)\right|^{2} \operatorname{div} \mathcal{G}\left(w^{1}\right)-\mu^{\prime}\left(\left|\mathbb{D}^{D} w^{1}\right|^{2}\right)\left|\mathbb{D}^{D} w^{1}\right|^{2} \operatorname{div} \mathbb{D}^{D} w^{1}\right. \\
& \left.-\mu^{\prime}\left(\left|\mathcal{G}\left(w^{2}\right)\right|^{2}\right)\left|\mathcal{G}\left(w^{2}\right)\right|^{2} \operatorname{div} \mathcal{G}\left(w^{2}\right)+\mu^{\prime}\left(\left|\mathbb{D}^{D} w^{2}\right|^{2}\right)\left|\mathbb{D}^{D} w^{2}\right|^{2} \operatorname{div} \mathbb{D}^{D} w^{2}\right) \\
& +\left(\lambda\left(\mathcal{D}\left(w^{1}\right)\right) \nabla \mathcal{D}\left(w^{1}\right)-\lambda\left(\operatorname{div} w^{1}\right) \nabla \operatorname{div} w^{1}\right. \\
& \left.+\lambda\left(\mathcal{D}\left(w^{2}\right)\right) \nabla \mathcal{D}\left(w^{2}\right)-\lambda\left(\operatorname{div} w^{2}\right) \nabla \operatorname{div} w^{2}\right) \\
& +\left(\lambda^{\prime}\left(\mathcal{D}\left(w^{1}\right)\right) \mathcal{D}\left(w^{1}\right) \nabla \mathcal{D}\left(w^{1}\right)-\lambda^{\prime}\left(\operatorname{div} w^{1}\right) \operatorname{div} w^{1} \nabla \operatorname{div} w^{1}\right. \\
& \left.-\lambda^{\prime}\left(\mathcal{D}\left(w^{2}\right)\right) \mathcal{D}\left(w^{2}\right) \nabla \mathcal{D}\left(w^{2}\right)+\lambda^{\prime}\left(\operatorname{div} w^{2}\right) \operatorname{div} w^{2} \nabla \operatorname{div} w^{2}\right)=\sum_{j=1}^{4} I_{j} .
\end{aligned}
$$

We estimate the norm of each $I_{j}$ separately. First, denoting

$$
\mathcal{G}_{s}^{j}=s \mathcal{G}\left(w^{j}\right)+(1-s) \mathbb{D}^{D} w^{j} j=1,2
$$


we obtain by (31), (33) and (34)

$$
\begin{aligned}
I_{1}= & -\int_{0}^{1}\left(\mu^{\prime}\left(\left|\mathcal{G}_{s}^{1}\right|^{2}\right)-\mu^{\prime}\left(\left|\mathcal{G}_{s}^{2}\right|^{2}\right)\right) \mathcal{G}_{s}^{1} \cdot \operatorname{sym}\left(\nabla w^{1} E_{w_{1}}\right)^{D} \operatorname{div} \mathcal{G}_{s}^{1} \\
& +\mu^{\prime}\left(\left|\mathcal{G}_{s}^{2}\right|^{2}\right)\left(\mathcal{G}_{s}^{1}-\mathcal{G}_{s}^{2}\right) \cdot \operatorname{sym}\left(\nabla w^{1} E_{w_{1}}\right)^{D} \operatorname{div} \mathcal{G}_{s}^{1} \\
& +\mu^{\prime}\left(\left|\mathcal{G}_{s}^{2}\right|^{2}\right) \mathcal{G}_{s}^{2} \cdot \operatorname{sym}\left(\nabla\left(w^{1}-w^{2}\right) E_{w^{1}}\right)^{D} \operatorname{div} \mathcal{G}_{s}^{1} \\
& +\mu^{\prime}\left(\left|\mathcal{G}_{s}^{2}\right|^{2}\right) \mathcal{G}_{s}^{2} \cdot \operatorname{sym}\left(\nabla w^{2} E_{w^{1}-w^{2}}\right)^{D} \operatorname{div} \mathcal{G}_{s}^{1} \\
& +\mu^{\prime}\left(\left|\mathcal{G}_{s}^{2}\right|^{2}\right) \mathcal{G}_{s}^{2} \cdot \operatorname{sym}\left(\nabla w^{2} E_{w^{2}}\right)^{D} \operatorname{div}\left(\mathcal{G}_{s}^{1}-\mathcal{G}_{s}^{2}\right) \\
& +\left(\mu\left(\left|\mathcal{G}_{s}^{1}\right|^{2}\right)-\mu\left(\left|\mathcal{G}_{s}^{2}\right|^{2}\right)\right) \operatorname{div} \operatorname{sym}\left(\nabla w^{1} E_{w^{1}}\right)^{D} \\
& +\mu\left(\left|\mathcal{G}_{s}^{2}\right|^{2}\right) \operatorname{div} \operatorname{sym}\left(\nabla\left(w^{1}-w^{2}\right) E_{w^{1}}\right)^{D} \\
& +\mu\left(\left|\mathcal{G}_{s}^{2}\right|^{2}\right) \operatorname{div} \operatorname{sym}\left(\nabla w^{2} E_{w^{1}-w^{2}}\right)^{D} \mathrm{~d} s
\end{aligned}
$$

and

$$
\begin{aligned}
I_{2}= & -\int_{0}^{1} 2\left(\left(\mu^{\prime \prime}\left(\left|\mathcal{G}_{s}^{1}\right|^{2}\right)-\mu^{\prime \prime}\left(\left|\mathcal{G}_{s}^{2}\right|^{2}\right)\right)\left|\mathcal{G}_{s}^{1}\right|^{2}+\mu^{\prime}\left(\left|\mathcal{G}_{s}^{1}\right|^{2}\right)-\mu^{\prime}\left(\left|\mathcal{G}_{s}^{2}\right|^{2}\right)\right) \\
& \mathcal{G}_{s}^{1} \cdot \operatorname{sym}\left(\nabla w^{1} E_{w^{1}}\right)^{D} \operatorname{div} \mathcal{G}_{s}^{1}+2 \mu^{\prime \prime}\left(\left|\mathcal{G}_{s}^{2}\right|^{2}\right)\left(\left|\mathcal{G}_{s}^{1}\right|^{2}-\left|\mathcal{G}_{s}^{2}\right|^{2}\right) \mathcal{G}_{s}^{1} \cdot \operatorname{sym}\left(\nabla w^{1} E_{w^{1}}\right)^{D} \operatorname{div} \mathcal{G}_{s}^{1} \\
& +2\left(\mu^{\prime \prime}\left(\left|\mathcal{G}_{s}^{2}\right|^{2}\right)\left|\mathcal{G}_{s}^{2}\right|^{2}+\mu^{\prime}\left(\left|\mathcal{G}_{s}^{2}\right|^{2}\right)\right)\left(\mathcal{G}_{s}^{1}-\mathcal{G}_{s}^{2}\right) \cdot \operatorname{sym}\left(\nabla w^{1} E_{w^{1}}\right)^{D} \operatorname{div} \mathcal{G}_{s}^{1} \\
& +2\left(\mu^{\prime \prime}\left(\left|\mathcal{G}_{s}^{2}\right|^{2}\right)\left|\mathcal{G}_{s}^{2}\right|^{2}+\mu^{\prime}\left(\left|\mathcal{G}_{s}^{2}\right|^{2}\right)\right) \mathcal{G}_{s}^{2} \cdot \operatorname{sym}\left(\nabla\left(w^{1}-w^{2}\right) E_{w^{1}}\right)^{D} \operatorname{div} \mathcal{G}_{s}^{1} \\
& +2\left(\mu^{\prime \prime}\left(\left|\mathcal{G}_{s}^{2}\right|^{2}\right)\left|\mathcal{G}_{s}^{2}\right|^{2}+\mu^{\prime}\left(\left|\mathcal{G}_{s}^{2}\right|^{2}\right)\right) \mathcal{G}_{s}^{2} \cdot \operatorname{sym}\left(\nabla w^{2} E_{w^{1}-w^{2}}\right)^{D} \operatorname{div} \mathcal{G}_{s}^{1} \\
& +2\left(\mu^{\prime \prime}\left(\left|\mathcal{G}_{s}^{2}\right|^{2}\right)\left|\mathcal{G}_{s}^{2}\right|^{2}+\mu^{\prime}\left(\left|\mathcal{G}_{s}^{2}\right|^{2}\right)\right) \mathcal{G}_{s}^{2} \cdot \operatorname{sym}\left(\nabla w^{2} E_{w^{2}}\right)^{D} \operatorname{div}\left(\mathcal{G}_{s}^{1}-\mathcal{G}_{s}^{2}\right) \\
& +\left(\mu\left(\left|\mathcal{G}_{s}^{1}\right|^{2}\right)-\mu\left(\left|\mathcal{G}_{s}^{2}\right|^{2}\right)\right)\left|\mathcal{G}_{s}^{1}\right|^{2} \operatorname{div} \operatorname{sym}\left(\nabla w^{1} E_{w^{1}}\right)^{D} \\
& +\mu\left(\left|\mathcal{G}_{s}^{2}\right|^{2}\right)\left(\left|\mathcal{G}_{s}^{1}\right|^{2}-\left|\mathcal{G}_{s}^{2}\right|^{2}\right) \operatorname{div} \operatorname{sym}\left(\nabla w^{1} E_{w^{1}}\right)^{D} \\
& +\mu\left(\left|\mathcal{G}_{s}^{2}\right|^{2}\right)\left|\mathcal{G}_{s}^{2}\right|^{2} \operatorname{div} \operatorname{sym}\left(\nabla\left(w^{1}-w^{2}\right) E_{w^{1}}\right)^{D} \\
& +\mu\left(\left|\mathcal{G}_{s}^{2}\right|^{2}\right)\left|\mathcal{G}_{s}^{2}\right|^{2} \operatorname{div} \operatorname{sym}\left(\nabla w^{2} E_{w^{1}-w^{2}}\right)^{D}
\end{aligned}
$$

Then denoting

$$
\mathcal{D}_{s}^{j}=s \mathcal{D}\left(w^{j}\right)+(1-s) \operatorname{div} w^{j} j=1,2
$$

we obtain using (36)

$$
\begin{aligned}
I_{3}= & -\int_{0}^{1}\left(\lambda^{\prime}\left(\mathcal{D}_{s}^{1}\right)-\lambda^{\prime}\left(\mathcal{D}_{s}^{2}\right)\right) \nabla w^{1} \cdot E_{w^{1}} \nabla \mathcal{D}_{s}^{1}+\lambda^{\prime}\left(\mathcal{D}_{s}^{2}\right) \nabla\left(w^{1}-w^{2}\right) \cdot E_{w^{1}} \nabla \mathcal{D}_{s}^{1} \\
& +\lambda^{\prime}\left(\mathcal{D}_{s}^{2}\right) \nabla w^{2} \cdot E_{w^{1}-w^{2}} \nabla \mathcal{D}_{s}^{1}+\lambda^{\prime}\left(\mathcal{D}_{s}^{2}\right) \nabla w^{2} \cdot E_{w^{2}} \nabla\left(\mathcal{D}_{s}^{1}-\mathcal{D}_{s}^{2}\right) \\
& +\left(\lambda\left(\mathcal{D}_{s}^{1}\right)-\lambda\left(\mathcal{D}_{s}^{2}\right)\right) \nabla\left(\nabla w^{1} \cdot E_{w^{1}}\right)+\lambda\left(\mathcal{D}_{s}^{2}\right) \nabla\left(\nabla\left(w^{1}-w^{2}\right) \cdot E_{w^{1}}\right) \\
& +\lambda\left(\mathcal{D}_{s}^{2}\right) \nabla\left(\nabla w^{2} \cdot E_{w^{1}-w^{2}}\right) \mathrm{d} s
\end{aligned}
$$

and

$$
\begin{aligned}
I_{4}= & -\int_{0}^{1}\left(\lambda^{\prime \prime}\left(\mathcal{D}_{s}^{1}\right)-\lambda^{\prime \prime}\left(\mathcal{D}_{s}^{2}\right)\right) \nabla w^{1} \cdot E_{w^{1}} \mathcal{D}_{s}^{1} \nabla \mathcal{D}_{s}^{1}+\lambda^{\prime \prime}\left(\mathcal{D}_{s}^{2}\right) \nabla\left(w^{1}-w^{2}\right) \cdot E_{w^{1}} \mathcal{D}_{s}^{1} \nabla \mathcal{D}_{s}^{1} \\
& +\lambda^{\prime \prime}\left(\mathcal{D}_{s}^{2}\right) \nabla w^{2} \cdot E_{w^{1}-w^{2}} \mathcal{D}_{s}^{1} \nabla \mathcal{D}_{s}^{1}+\lambda^{\prime \prime}\left(\mathcal{D}_{s}^{2}\right) \nabla w^{2} \cdot E_{w^{2}}\left(\mathcal{D}_{s}^{1}-\mathcal{D}_{s}^{2}\right) \nabla \mathcal{D}_{s}^{1} \\
& +\lambda^{\prime \prime}\left(\mathcal{D}_{s}^{2}\right) \nabla w^{2} \cdot E_{w^{2}} \mathcal{D}_{s}^{2} \nabla\left(\mathcal{D}_{s}^{1}-\mathcal{D}_{s}^{2}\right)+\left(\lambda^{\prime}\left(\mathcal{D}_{s}^{1}\right)-\lambda^{\prime}\left(\mathcal{D}_{s}^{2}\right)\right) \mathcal{D}_{s}^{1} \nabla\left(\nabla w^{1} \cdot E_{w^{1}}\right) \\
& +\lambda^{\prime}\left(\mathcal{D}_{s}^{2}\right)\left(\mathcal{D}_{s}^{1}-\mathcal{D}_{s}^{2}\right) \nabla\left(\nabla w^{1} \cdot E_{w^{1}}\right)+\lambda^{\prime}\left(\mathcal{D}_{s}^{2}\right) \mathcal{D}_{s}^{2} \nabla\left(\nabla\left(w^{1}-w^{2}\right) \cdot E_{w^{1}}\right) \\
& +\lambda^{\prime}\left(\mathcal{D}_{s}^{2}\right) \mathcal{D}_{s}^{2} \nabla\left(\nabla w^{2} \cdot E_{w^{1}-w^{2}}\right) \mathrm{d} s .
\end{aligned}
$$


We also have the estimates

$$
\begin{aligned}
& \left\|\mathcal{G}_{s}^{1}-\mathcal{G}_{s}^{2}\right\|_{L^{\infty}\left(0, T ; L^{\infty}(\Omega)\right)} \\
& \quad \leq\left\|\mathbb{D}^{D}\left(w^{1}-w^{2}\right)\right\|_{L^{\infty}\left(0, T ; L^{\infty}(\Omega)\right)}+\left\|\nabla\left(w^{1}-w^{2}\right)\right\|_{L^{\infty}\left(0, T ; L^{\infty}(\Omega)\right)}\left\|E_{w^{1}}\right\|_{L^{\infty}\left(0, T ; L^{\infty}(\Omega)\right)} \\
& \quad+\left\|\nabla w^{2}\right\|_{L^{\infty}\left(0, T ; L^{\infty}(\Omega)\right)}\left\|E_{w^{1}-w^{2}}\right\|_{L^{\infty}\left(0, T ; L^{\infty}(\Omega)\right)} \\
& \quad \leq c\left\|w^{1}-w^{2}\right\|_{\mathcal{V}^{p, q}\left(Q_{T}\right)}\left(1+T^{\frac{1}{p^{\prime}}}\left(\left\|w^{1}\right\|_{L^{p}\left(0, T ; W^{2, q}(\Omega)\right)}+\left\|w^{2}\right\|_{\mathcal{V}^{p, q}\left(Q_{T}\right)}\right)\right)
\end{aligned}
$$

and

$$
\begin{aligned}
& \left\|\operatorname{div}\left(\mathcal{G}_{s}^{1}-\mathcal{G}_{s}^{2}\right)\right\|_{L^{p}\left(0, T ; L^{q}(\Omega)\right)} \\
& \leq c \\
& \quad\left\|\nabla^{2} w^{1}-w^{2}\right\|_{L^{p}\left(0, T ; L^{q}(\Omega)\right)}\left(1+\left\|E_{w^{2}}\right\|_{L^{\infty}\left(0, T ; L^{\infty}(\Omega)\right)}\right) \\
& \quad+c\left\|\nabla\left(w^{1}-w^{2}\right)\right\|_{L^{\infty}\left(0, T ; L^{\infty}(\Omega)\right)}\left\|\nabla E_{w^{2}}\right\|_{L^{p}\left(0, T ; L^{q}(\Omega)\right)} \\
& \quad+\left\|\nabla^{2} w^{1}\right\|_{L^{p}\left(0, T ; L^{q}(\Omega)\right)}\left\|E_{w^{2}-w^{1}}\right\|_{L^{\infty}\left(0, T ; L^{\infty}(\Omega)\right)} \\
& \quad+\left\|\nabla w^{1}\right\|_{L^{\infty}\left(0, T ; L^{\infty}(\Omega)\right)}\left\|\nabla E_{w^{2}-w^{1}}\right\|_{L^{p}\left(0, T ; L^{q}(\Omega)\right)} \\
& \leq c
\end{aligned}
$$

by (23) and (21). By the previous two estimates along with (401), (41), (42), (45) and (46) in straightforward manipulations similar to the ones in the boundedness proof we infer that

$$
\sum_{j=1}^{4}\left\|I_{j}\right\|_{L^{p}\left(0, T ; L^{q}(\Omega)\right)} \leq\left\|w^{1}-w^{2}\right\|_{\mathcal{V}^{p, q}\left(Q_{T}\right)} c K \sum_{j=1}^{\tilde{N}} M^{p_{j}} L^{r_{j}} T^{\kappa_{j}}, p_{j}, r_{j} \in \mathbf{N} \cup\{0\}, \kappa_{j}>0 .
$$

We omit the detailed presentation of the proof of the latter inequality in order to keep the length of the paper in a reasonable limit.

We infer in a straightforward way

$$
\begin{aligned}
\left\|\vartheta^{1} \partial_{t} w^{1}-\vartheta^{2} \partial_{t} w^{2}\right\|_{L^{p}\left(0, T ; L^{q}(\Omega)\right)} \leq & \left\|\vartheta^{1}-\vartheta^{2}\right\|_{L^{\infty}\left(0, T ; L^{\infty}(0,)\right)}\left\|\partial_{t} w^{1}\right\|_{L^{p}\left(0, T ; L^{q}(\Omega)\right)} \\
& +\left\|\vartheta^{2}\right\|_{L^{\infty}\left(0, T ; L^{\infty}(0,)\right)}\left\|\partial_{t} w^{1}-\partial_{t} w^{2}\right\|_{L^{p}\left(0, T ; L^{q}(\Omega)\right)} \\
\leq & c T^{\frac{1}{p^{p}}} M\left(\left\|\vartheta^{1}-\vartheta^{2}\right\|_{W^{1, p}\left(0, T ; W^{1, q}(\Omega)\right)}+\left\|\partial_{t}\left(w^{1}-w^{2}\right)\right\|_{L^{p}\left(0, T ; L^{q}(\Omega)\right)}\right)
\end{aligned}
$$

and

$$
\begin{aligned}
& \left\|\left(\pi^{\prime}\left(\rho_{*}+\theta_{0}+\vartheta^{1}\right)-\pi^{\prime}\left(\rho_{*}+\theta_{0}\right)\right) \nabla\left(\theta_{0}+\vartheta^{1}\right)-\left(\pi^{\prime}\left(\rho_{*}+\theta_{0}+\vartheta^{2}\right)-\pi^{\prime}\left(\rho_{*}+\theta_{0}\right)\right) \nabla\left(\theta_{0}+\vartheta^{2}\right)\right\|_{L^{p}\left(0, T ; L^{q}(\Omega)\right)} \\
& \leq \Pi\left\|\vartheta^{1}-\vartheta^{2}\right\|_{L^{\infty}\left(0, T ; L^{\infty}(\Omega)\right)}\left\|\theta_{0}+\vartheta^{1}\right\|_{L^{p}\left(0, T ; W^{1, q}(\Omega)\right)}+2 \Pi\left\|\vartheta^{1}-\vartheta^{2}\right\|_{L^{p}\left(0, T ; W^{1, q}(\Omega)\right)} \\
& \leq c \Pi T^{\frac{1}{p^{\prime}}}(L+M+1)\left\|\vartheta^{1}-\vartheta^{2}\right\|_{W^{1, p}\left(0, T ; W^{1, q}(\Omega)\right)} .
\end{aligned}
$$


Applying (38) and (21) we have

$$
\begin{aligned}
& \left\|\operatorname{div}\left(\mathcal{S}\left(\mathbb{D}^{D} w^{1}\right)-\mathcal{S}\left(\mathbb{D}^{D} w^{2}\right)\right)-\mathcal{A}\left(\mathbb{D} u_{0}\right)\left(\mathbb{D}\left(w^{1}-w^{2}\right)\right)\right\|_{L^{p}\left(0, T ; L^{q}(\Omega)\right)} \\
= & \left\|\mathcal{A}\left(\mathbb{D} w^{1}\right)\left(\mathbb{D} w^{1}\right)-\mathcal{A}\left(\mathbb{D} w^{2}\right)\left(\mathbb{D} w^{2}\right)-\mathcal{A}\left(\mathbb{D} u_{0}\right)\left(\mathbb{D}\left(w^{1}-w^{2}\right)\right)\right\|_{L^{p}\left(0, T ; L^{q}(\Omega)\right)} \\
\leq & \left\|\mathcal{A}\left(\mathbb{D} w^{1}\right)\left(\mathbb{D}\left(w^{1}-w^{2}\right)\right)-\mathcal{A}\left(\mathbb{D} u_{0}\right)\left(\mathbb{D}\left(w^{1}-w^{2}\right)\right)\right\|_{L^{p}\left(0, T ; L^{q}(\Omega)\right)} \\
& +\left\|\mathcal{A}\left(\mathbb{D} w^{1}\right)\left(\mathbb{D} w^{2}\right)-(A)\left(\mathbb{D} w^{2}\right)\left(\mathbb{D} w^{2}\right)\right\|_{L^{p}\left(0, T ; L^{q}(\Omega)\right)} \\
\leq & \sum_{j, k, l, m=1}^{d}\left(\left\|a_{j k}^{l m}\left(\mathbb{D} w^{1}\right)-a_{j k}^{l m}\left(\mathbb{D} u_{0}\right)\right\|_{L^{\infty}\left(0, T ; L^{\infty}(\Omega)\right)}\left\|\partial_{k} \partial_{l}\left(w^{1}-w^{2}\right)\right\|_{L^{p}\left(0, T ; L^{q}(\Omega)\right)}\right. \\
& \left.+\left\|a_{j k}^{l m}\left(\mathbb{D} w^{1}\right)-a_{j k}^{l m}\left(\mathbb{D} w^{2}\right)\right\|_{L^{\infty}\left(0, T ; L^{\infty}(\Omega)\right)}\left\|\partial_{k} \partial_{l} w^{2}\right\|_{L^{p}\left(0, T ; L^{q}(\Omega)\right)}\right) \\
\leq & c K\left(1+\left\|w^{1}\right\|_{L^{\infty}\left(0, W^{1, \infty}(\Omega)\right)}+\left\|u_{0}\right\|_{W^{1, \infty}(\Omega)}+\left\|w^{1}\right\|_{L^{\infty}\left(0, W^{1, \infty}(\Omega)\right)}^{2}+\left\|u_{0}\right\|_{W^{1, \infty}(\Omega)}\right) \\
& \times\left\|w^{1}-u_{0}\right\|_{L^{\infty}\left(0, T ; W^{1, \infty}(\Omega)\right)}\left\|w^{1}-w^{2}\right\|_{L^{p}\left(0, T ; W^{2, q}(\Omega)\right)} \\
& +c K\left(1+\left\|w^{1}\right\|_{L^{\infty}\left(0, W^{1, \infty}(\Omega)\right)}+\left\|w^{2}\right\|_{W^{1, \infty}(\Omega)}+\left\|w^{1}\right\|_{L^{\infty}\left(0, W^{1, \infty}(\Omega)\right)}^{2}+\left\|w^{2}\right\|_{W^{1, \infty}(\Omega)}\right) \\
& \times\left\|w^{1}-w^{2}\right\|_{L^{\infty}\left(0, T ; W^{1, \infty}(\Omega)\right)}\left\|w^{2}\right\|_{L^{p}\left(0, T ; W^{2, q}(\Omega)\right)} \\
\leq & c K\left(1+(M+L)+L+(M+L)^{2}+L^{2}\right) M\left\|w^{1}-w^{2}\right\|_{\mathcal{V}^{p, q}\left(Q_{T}\right)} \\
& +c K(M+L)\left(1+(M+L)+(M+L)^{2}\right)\left\|w^{1}-w^{2}\right\|_{\mathcal{V}^{p, q}\left(Q_{T}\right)} .
\end{aligned}
$$

Taking into account (56), (57), (58), (59), (60), (61), (62) and (63) we conclude

$$
\begin{aligned}
& \left\|\mathcal{G}\left(\vartheta^{1}, w^{1}\right)-\mathcal{G}\left(\vartheta^{2}, w^{2}\right)\right\|_{L^{p}\left(0, T ; W^{1, q}(\Omega)\right)}+\left\|\mathcal{F}\left(\vartheta^{1}, w^{1}\right)-\mathcal{F}\left(\vartheta^{2}, w^{2}\right)\right\|_{L^{p}\left(0, T ; L^{q}(\Omega)\right)} \\
& \leq\left[\vartheta^{1}-\vartheta^{2}, w^{1}-w^{2}\right]_{T, M} c\left(\sum_{j=1}^{Q_{1}} K^{k_{j}} L^{l_{j}} M^{m_{j}} \Pi^{\left|k_{j}-1\right|} T^{\beta_{j}}+\sum_{j=1}^{Q_{1}} K^{n_{j}} L^{o_{j}} M^{p_{j}}\right),
\end{aligned}
$$

where $l_{j}, m_{j}, o_{j} \in \mathbf{N} \cup\{0\}, \beta_{j}>0, p_{j} \in \mathbf{N} \backslash\{1\}$ and $k_{j}, n_{i} \in\{0,1\}$.

As before, $T$ and $M$ can be chosen such that the sum in the bracket on the right hand side is less than $c$ for arbitrary $c>0$. Consequently, $T$ and $M$ can be chosen such that $\Phi$ is a contraction according to Theorem 3.1 and the Banach fix-point theorem then yields the validity of Theorem 1.1.

It only remains to prove Theorem 3.1. This is content of the next section.

\section{$3 \quad L^{p}$ regularity of the linearized system}

The main aim of this section is to proof the following regularity theorem for (19). For simplicity, we denote $u=\tilde{u}-u_{0}$ throughout this section.

Theorem 3.1. Let $\theta_{0}, \nabla u_{0} \in B U C(\Omega)^{1}$ and let $\varrho_{*}+\theta_{0} \in L^{\infty}(\Omega)$ and $\left(\varrho_{*}+\theta_{0}\right)^{-1} \in L^{\infty}(\Omega)$. Then for every $(\mathcal{G}, \mathcal{F}) \in L^{p}\left(0, T ; W^{1, q}(\Omega)\right) \times L^{p}\left(0, T ; L^{q}(\Omega)\right)$ the solution $(\theta, u)$ to (19) satisfies

$$
\|u\|_{\mathcal{V}^{p, q}\left(Q_{T}\right)}+\|\theta\|_{W^{1, p}\left(0, T ; W^{1, q}(\Omega)\right)} \leq c\left(\|\mathcal{G}\|_{L^{p}\left(0, T ; W^{1, q}(\Omega)\right)}+\|\mathcal{F}\|_{L^{p}\left(0, T ; L^{q}(\Omega)\right.}\right)
$$

where $c$ is a constant independent of $\theta, \tilde{u}$ and the right hand side.

\footnotetext{
${ }^{1}$ Here $B U C$ denotes the space of bounded and uniformly continuous functions. Note that $W^{1, q}(\Omega) \hookrightarrow B U C(\Omega)$ whenever $q>d$.
} 
In order to reach this goal we use a semi-group approach which may be found in e.g. [12] or in [13. In particular, the equation (19) can be rewritten as

$$
\partial_{t}\left(\begin{array}{l}
\theta \\
u
\end{array}\right)-\mathcal{A}\left(\begin{array}{l}
\theta \\
u
\end{array}\right)=\left(\begin{array}{l}
\mathcal{G} \\
\mathcal{F}
\end{array}\right)
$$

for $\mathcal{A}$ defined as

$$
\mathcal{A}\left(\begin{array}{l}
\theta \\
u
\end{array}\right)=\left(\begin{array}{c}
-\left(\varrho_{*}+\theta_{0}\right) \operatorname{div} u \\
\frac{1}{\varrho_{*}+\theta_{0}} \mathcal{A}\left(\mathbb{D} u_{0}\right)(\mathbb{D} u)-\frac{1}{\varrho_{*}+\theta_{0}} \pi^{\prime}\left(\varrho_{*}+\theta_{0}\right) \nabla \theta^{\cdot}
\end{array}\right)
$$

The equation (64) is equipped with zero initial conditions, i.e. $\theta(0, \cdot) \equiv 0$ and $u(0, \cdot) \equiv 0$. Note that here we write $\mathcal{F}$ instead of $\frac{1}{\varrho_{*}+\theta_{0}} \mathcal{F}$. We can afford this inaccuracy since $\frac{1}{\varrho_{*}+\theta_{0}}$ and $\varrho_{*}+\theta_{0}$ are bounded in $L^{\infty}$ in all applications and thus

$$
\frac{1}{\varrho_{*}+\theta_{0}} \mathcal{F} \in L^{p}\left(0, T ; L^{q}(\Omega)\right) \Leftrightarrow \mathcal{F} \in L^{p}\left(0, T ; L^{q}(\Omega)\right) .
$$

The resolvent problem for such equation is

$$
\lambda\left(\begin{array}{l}
\theta \\
u
\end{array}\right)-\mathcal{A}\left(\begin{array}{l}
\theta \\
u
\end{array}\right)=\left(\begin{array}{l}
\mathcal{G} \\
\mathcal{F}
\end{array}\right) .
$$

First, we recall the definition of $\mathcal{R}$-boundedness (see [13, Definition 2.1]):

Definition 3.1. A family $\mathcal{T} \subset \mathcal{L}(X, Y)$ (i.e. a family of linear operators from $X$ to $Y$ ) is called $\mathcal{R}$-bounded if there exist constants $C>0$ and $p \in[1, \infty)$ such that for each $n \in \mathbb{N}, T_{j} \in \mathcal{T}$, $f_{j} \in X\left(j \in\{1, \ldots, n\}\right.$ and for all sequences $\left\{r_{j}(v)\right\}_{j=1}^{n}$ of independent, symmetric, $\{-1,1\}$-valued random variables on $[0,1]$ there holds

$$
\int_{0}^{1}\left\|\sum_{j=1}^{n} r_{j}(v) T_{j} f_{j}\right\|^{p} \mathrm{~d} v \leq C \int_{0}^{1}\left\|\sum_{j=1}^{n} r_{j}(v) f_{j}\right\|_{X}^{p} \mathrm{~d} v .
$$

The smallest such $C$ is calles $\mathcal{R}$-bound and it is denoted by $\mathcal{R}_{\mathcal{L}(X, Y)}(\mathcal{T})$.

We aim to prove the $\mathcal{R}$-sectoriality of the operator $\mathcal{A}$, i.e., to justify that $\mathcal{R}_{\lambda}=\lambda\left(\lambda \mathbb{I}_{d}-\mathcal{A}\right)^{-1}$ is $\mathcal{R}$-bounded for all $\lambda \in \Sigma_{\beta, \nu}$ for some $\beta \in\left(0, \frac{\pi}{2}\right)$ and $\nu>0$. Here

$$
\Sigma_{\beta, \nu}=\{\mu \in \mathbb{C}, \arg (\mu-\nu) \leq \pi-\beta\} .
$$

In the last part of this section we show that this $\mathcal{R}$-boundedness is sufficient for the $L^{p}-L^{q}$ regularity. To reach this goal, we use the following theorem which is due to Weis.

Theorem 3.2. Let $X$ and $Y$ be two $U M D$ spaces and $p \in(1, \infty)$. Let $M$ be a function in $C^{1}(\mathbb{R} \backslash 0, \mathcal{L}(X, Y))$ such that

$$
\begin{aligned}
& \mathcal{R}_{\mathcal{L}(X, Y)}(\{M(\tau), \tau \in \mathbb{R} \backslash\{0\}\})=\kappa_{0}<\infty \\
& \mathcal{R}_{\mathcal{L}(X, Y)}\left(\left\{\tau M^{\prime}(\tau), \tau \in \mathbb{R} \backslash\{0\}\right\}\right)=\kappa_{1}<\infty .
\end{aligned}
$$

Then $T_{M}$ defined as $T_{M} \Phi=\mathcal{F}^{-1}[M \mathcal{F}(\Phi)]$ is extended to a bounded linear operator from $L^{p}(\mathbb{R}, X)$ into $L^{p}(\mathbb{R}, Y)$. Moreover,

$$
\left\|T_{M}\right\|_{\mathcal{L}(X, Y)} \leq c\left(\kappa_{0}+\kappa_{1}\right)
$$

where $c>0$ is independent of $p, X, Y, \kappa_{0}$ and $\kappa_{1}$. 
Here $U M D$ stands for spaces with uniform martingale differences. Their properties might be found in [8] or in 31. For our purposes it is enough to know that $L^{p}$ and $W^{k, p}$ are $U M D$ once $p \in(1, \infty)$.

Since we assume $\lambda \neq 0$ in (65)

$$
\theta=\frac{1}{\lambda}\left(\mathcal{G}-\left(\varrho_{*}+\theta_{0}\right) \operatorname{div} u\right) .
$$

Consequently, we may rewrite the resolvent problem as

$$
\lambda u-\mathcal{B}_{\lambda}(u)=F_{\lambda} \quad \text { on } \Omega
$$

where

$$
\begin{aligned}
& \mathcal{B}_{\lambda}(u)=\frac{1}{\varrho_{*}+\theta_{0}} \mathcal{A}\left(\mathbb{D} u_{0}\right)(\mathbb{D} u)+\pi^{\prime}\left(\varrho_{*}+\theta_{0}\right) \frac{1}{\lambda} \nabla \operatorname{div} u \\
& F_{\lambda}=T_{\lambda}\left(\begin{array}{c}
\mathcal{G} \\
\mathcal{F}
\end{array}\right):=\left(\mathcal{F}-\nabla \mathcal{G} \frac{1}{\lambda}\right) .
\end{aligned}
$$

We claim the following.

Theorem 3.3. Let $\mathcal{U}_{\lambda}: L^{q}(\Omega) \mapsto W^{2, q}(\Omega)$ be a solution operator to (66). Then

$$
\begin{array}{r}
\mathcal{R}_{L^{q}(\Omega)}\left(\left\{\lambda \mathcal{U}_{\lambda}, \lambda \in \Sigma_{\beta, \nu}\right\}\right) \leq c \\
\mathcal{R}_{L^{q}(\Omega)}\left(\left\{|\lambda|^{1 / 2} \partial_{j} \mathcal{U}_{\lambda}, \lambda \in \Sigma_{\beta, \nu}\right\}\right) \leq c \\
\mathcal{R}_{L^{q}(\Omega)}\left(\left\{\partial_{j} \partial_{k} \mathcal{U}_{\lambda}, \lambda \in \Sigma_{\beta, \nu}\right\}\right) \leq c
\end{array}
$$

for every $j, k \in\{1, \ldots, d\}$, every $q \in(1, \infty)$ and for some $\beta \in(0, \pi / 2)$ and $\nu>0$.

Note that the $\mathcal{R}$-boundedness of $\mathcal{R}_{\lambda}$ is a consequence of this Theorem and Proposition 4.2 , Indeed, recall that $\mathcal{R}_{\lambda}$ has two components. In particular, $\mathcal{R}_{\lambda 1}\left(\begin{array}{c}\mathcal{G} \\ \mathcal{F}\end{array}\right)=\lambda \theta$ and $\mathcal{R}_{\lambda 2}\left(\begin{array}{l}\mathcal{G} \\ \mathcal{F}\end{array}\right)=\lambda u$. Both of them can be written as a composition and sum of certain operators, namely

$$
\begin{aligned}
& \mathcal{R}_{\lambda 1}\left(\begin{array}{c}
\mathcal{G} \\
\mathcal{F}
\end{array}\right)=\left(-\left(\varrho_{*}+\theta_{0}\right) \operatorname{div} \mathcal{U}_{\lambda} \circ T_{\lambda}\left(\begin{array}{c}
\mathcal{G} \\
\mathcal{F}
\end{array}\right)+\mathcal{G}\right) \\
& \mathcal{R}_{\lambda 2}\left(\begin{array}{c}
\mathcal{G} \\
\mathcal{F}
\end{array}\right)=\left(\lambda \mathcal{U}_{\lambda} \circ T_{\lambda}\right)\left(\begin{array}{c}
\mathcal{G} \\
\mathcal{F}
\end{array}\right) .
\end{aligned}
$$

Proposition 4.2 then yields the claim.

In order to show Theorem 3.3 we first prove its version with constant coefficients.

Theorem 3.4. Let $\mathcal{B}_{\lambda}(u): W^{2, q}\left(\mathbb{R}^{d}\right) \mapsto L^{q}\left(\mathbb{R}^{d}\right)$ be an operator defined as

$$
\mathcal{B}_{\lambda}(u)=\frac{1}{\gamma_{1}} \sum_{k, l, m=1}^{d} a_{j k}^{l m}(|D|) \partial_{l} \partial_{m} u_{k}+\frac{\gamma_{2}}{\lambda \gamma_{1}} \sum_{l=1}^{d} \partial_{j} \partial_{l} u_{l}
$$

where $a$ is defined by (44) and $D \in \mathbb{R}_{\mathrm{sym}}^{d \times d}, \gamma_{1}, \gamma_{2} \in \mathbb{R}^{+}$are arbitrary. Then the solution operator $\mathcal{U}_{\lambda}: L^{q}\left(\mathbb{R}^{d}\right) \mapsto W^{2, q}\left(\mathbb{R}^{d}\right), \mathcal{U}_{\lambda}: f \mapsto u$ where $u$ solves

$$
\lambda u-\mathcal{B}_{\lambda} u=f
$$


in $\mathbb{R}^{d}$ fulfills

$$
\begin{array}{r}
\mathcal{R}_{L^{q}\left(\mathbb{R}^{d}\right)}\left(\left\{\lambda \mathcal{U}_{\lambda}, \lambda \in \Sigma_{\beta, \nu}\right\}\right) \leq c \\
\mathcal{R}_{L^{q}\left(\mathbb{R}^{d}\right)}\left(\left\{|\lambda|^{1 / 2} \partial_{j} \mathcal{U}_{\lambda}, \lambda \in \Sigma_{\beta, \nu}\right\}\right) \leq c \\
\mathcal{R}_{L^{q}\left(\mathbb{R}^{d}\right)}\left(\left\{\partial_{j} \partial_{k} \mathcal{U}_{\lambda}, \lambda \in \Sigma_{\beta, \nu}\right\}\right) \leq c
\end{array}
$$

for every $j, k \in\{1, \ldots, d\}$, every $q \in(1, \infty)$ and for some $\beta \in(0, \pi / 2)$ and $\nu>0$.

Proof. We apply Fourier transform to rewrite (3.4) as

$$
\lambda \hat{u}_{m}+\frac{1}{\gamma_{1}} \sum_{j, k, l=1}^{d} a_{m j}^{k l}(|D|) \xi_{k} \xi_{l} \hat{u}_{j}+\frac{\gamma_{2}}{\lambda} \xi_{m} \sum_{l=1}^{d} \xi_{l} \hat{u}_{l}=\hat{f}_{m}
$$

where

$$
\hat{u}(\xi):=\mathcal{F}(u)=\int_{\mathbb{R}^{d}} e^{-i \eta \xi} u(\eta) \mathrm{d} \eta .
$$

Set

$$
\begin{aligned}
E(\xi)_{m j} & =\sum_{k, l=1}^{d} \frac{1}{\gamma_{1}} a_{m j}^{k l}(|D|) \xi_{k} \xi_{l} \\
E(\xi, \lambda)_{m l} & =\frac{\gamma_{2}}{\lambda \gamma_{1}} \xi_{m} \xi_{l} .
\end{aligned}
$$

Naturally,

$$
u=\mathcal{F}^{-1}\left(\left(\lambda \mathbb{I}_{d}+E(\xi)+E(\lambda, \xi)\right)^{-1} \hat{f}\right)
$$

assuming $\left(\lambda \mathbb{I}_{d}+E(\xi)+E(\lambda, \xi)\right)^{-1}$ exists - this is discussed below. Further,

$$
\begin{aligned}
\lambda u & =\mathcal{F}^{-1}\left(\lambda\left(\lambda \mathbb{I}_{d}+E(\xi)+E(\lambda, \xi)\right)^{-1} \hat{f}\right) \\
|\lambda|^{\frac{1}{2}} \partial_{j} u & =\mathcal{F}^{-1}\left(|\lambda|^{\frac{1}{2}} i \xi_{j}\left(\lambda \mathbb{I}_{d}+E(\xi)+E(\lambda, \xi)\right)^{-1} \hat{f}\right) \\
\partial_{j} \partial_{k} u & =\mathcal{F}^{-1}\left(-\xi_{j} \xi_{k}\left(\lambda \mathbb{I}_{d}+E(\xi)+E(\lambda, \xi)\right)^{-1} \hat{f}\right)
\end{aligned}
$$

According to Theorem 4.1] it suffices to show that the multipliers appearing in (67) satisfies

$$
\left|\partial_{\xi}^{\alpha} m(\lambda, \xi)\right| \leq c_{\alpha}|\xi|^{-|\alpha|}
$$

for all multi-indeces $\alpha \in \mathbb{N}_{0}^{d}$ and for all $\lambda \in \Sigma_{\beta, \nu}$ where $\beta$ and $\nu$ are appropriately chosen.

First, consider a matrix $\lambda \mathbb{I}_{d}+E(\xi)$. Since $E$ is elliptic and symmetric (see (8) and (5)) there is an orthogonal $\xi$-dependent matrix $O$ such that

$$
E(\xi)=O\left(\begin{array}{ccc}
\mu_{1} & 0 & 0 \\
0 & \mu_{2} & 0 \\
0 & 0 & \mu_{3}
\end{array}\right) O^{T} .
$$

We restrict ourselves here to the case of $3 \times 3$-matrices for clarity of the presentation. We consider without loss of generality $\mu_{3} \geq \mu_{2} \geq \mu_{1} \geq c|\xi|^{2}$ (here $c$ in fact depend on $D$, however, we assume in applications that $D$ is always bounded by some constant dependent on $\left.u_{0}\right)$. Consequently,

$$
\lambda \mathbb{I}_{d}+E(\xi)=O\left(\begin{array}{ccc}
\lambda+\mu_{1} & 0 & 0 \\
0 & \lambda+\mu_{2} & 0 \\
0 & 0 & \lambda+\mu_{3}
\end{array}\right) O^{T}
$$


and there exists $\left(\lambda \mathbb{I}_{d}+E(\xi)\right)^{-1}$ which may be written as

$$
\left(\lambda \mathbb{I}_{d}+E(\xi)\right)^{-1}=O\left(\begin{array}{ccc}
\frac{1}{\lambda+\mu_{1}} & 0 & 0 \\
0 & \frac{1}{\lambda+\mu_{2}} & 0 \\
0 & 0 & \frac{1}{\lambda+\mu_{3}}
\end{array}\right) O^{T} .
$$

We use Lemma 4.1 and the orthogonality of $O$ to claim

$$
\left|\left(\lambda \mathbb{I}_{d}+E(\xi)\right)^{-1}\right| \leq \frac{c(\nu)}{|\lambda|+|\xi|^{2}}
$$

in an operator norm. Further

$$
\left(\lambda \mathbb{I}_{d}+E(\xi)+E(\xi, \lambda)\right)^{-1}=\left(\lambda \mathbb{I}_{d}+E(\xi)\right)^{-1}\left(\mathbb{I}_{d}+\left(\lambda \mathbb{I}_{d}+E(\xi)\right)^{-1} E(\xi, \lambda)\right)^{-1}
$$

and, from definition,

$$
\left|\left(\lambda \mathbb{I}_{d}+E(\xi)\right)^{-1} E(\xi, \lambda)\right| \leq \frac{\gamma_{2}}{|\lambda|} \frac{c(\nu)|\xi|^{2}}{|\lambda|+c|\xi|^{2}} \leq \frac{1}{2}
$$

assuming $|\lambda|$ is sufficiently high. This can be managed by a proper choice of $\nu$ and $\beta$. For this choice we get $\lambda \mathbb{I}_{d}+E(\xi)+E(\xi, \lambda)$ invertible and

$$
\left|\left(\lambda \mathbb{I}_{d}+E(\xi)+E(\xi, \lambda)\right)^{-1}\right| \leq \frac{c(\nu)}{|\lambda|+|\xi|^{2}}
$$

To proceed further, we prove the following lemma:

Lemma 3.1. Let $|\lambda|>\lambda_{0}>0$. There exist coefficients $a_{j, n}$ depending on $\lambda_{0}$ such that

$$
\left|\partial_{\xi}^{\alpha}\left(\lambda \mathbb{I}_{d}+E(\xi)+E(\xi, \lambda)\right)^{-1}\right| \leq \sum_{j=0}^{\left\lfloor\frac{n}{2}\right\rfloor} a_{j, n}|\xi|^{n-2 j}\left(\frac{c(\nu)}{|\lambda|+|\xi|^{2}}\right)^{1+n-j}
$$

for all multi-indeces $\alpha$ with $|\alpha|=n$

Proof. During this proof, we assume that all matrix multiplications appearing in the proof are commutative. This allows to simplify the notation and it does not affect the validity of the main claim. Using this assumption, we are going to prove

$$
\partial_{\xi}^{\alpha}\left(\lambda \mathbb{I}_{d}+E(\xi)+E(\xi, \lambda)\right)^{-1}=\sum_{j=0}^{\left\lfloor\frac{n}{2}\right\rfloor} a_{j, n}\left(E^{\prime}(\xi)+E^{\prime}(\xi, \lambda)\right)^{n-2 j}(\lambda \mathbb{I}+E(\xi)+E(\xi, \lambda))^{-1-n+j}
$$

Here we also use another simplification as $E^{\prime}(\xi)$ denotes arbitrary first partial derivative and the bracket $\left(E^{\prime}(\xi)+E^{\prime}(\xi, \lambda)\right)^{n-2 j}$ should be read as the product of $n-2 j$ first derivatives which are not necessarily with respect to the same coordinate. We also assume that $a_{j, n}$ is independent of $\lambda$ as far as $|\lambda|$ is far away from zero.

Clearly, (69) follows directly from (70). We proof (170) by induction. Let $n=0$, then

$$
\partial^{0}\left(\lambda \mathbb{I}_{d}+E(\xi)+E(\xi, \lambda)\right)^{-1}=\left(\lambda \mathbb{I}_{d}+E(\xi)+E(\xi, \lambda)\right)^{-1}
$$


Now let (70) holds for certain $n \in \mathbb{N}$ and let $\alpha$ be a multiindex of length $|\alpha|=n+1$. Then

$$
\partial_{\xi}^{\alpha}\left(\lambda \mathbb{I}_{d}+E(\xi)+E(\xi, \lambda)\right)^{-1}=\left(\partial_{\xi}^{\alpha^{\prime}}\left(\lambda \mathbb{I}_{d}+E(\xi)+E(\xi, \lambda)\right)^{-1}\right)^{\prime}
$$

for certain multi-index $\alpha^{\prime}$ with $\left|\alpha^{\prime}\right|=n$. Since $E(\xi)$ and $E(\xi, \lambda)$ are second order polynomials in $\xi, E^{\prime \prime}(\xi)$ and $E^{\prime \prime}(\xi, \lambda)$ are constants which might be included into $a_{j, n}$. We apply one partial derivation on the right hand side of (70). For $j \neq \frac{n}{2}$ we get (throughout this proof we use the letter $a$ to denote a constant which may vary from line to line but it possesses the same dependencies as $\left.a_{j, n}\right)$

$$
\begin{aligned}
& \left(\left(E^{\prime}(\xi)+E^{\prime}(\xi, \lambda)\right)^{n-2 j}\left(\lambda \mathbb{I}_{d}+E(\xi)+E(\xi, \lambda)\right)^{-1-n+j}\right)^{\prime}= \\
& \begin{aligned}
a\left(E^{\prime}(\xi)+E^{\prime}(\xi, \lambda)\right)^{n-2 j-1}\left(\lambda \mathbb{I}_{d}+E(\xi)+E(\xi, \lambda)\right)^{-1-n+j} \\
+a\left(E^{\prime}(\xi)+E^{\prime}(\xi, \lambda)\right)^{n-2 j+1}\left(\lambda \mathbb{I}_{d}+E(\xi)+E(\xi, \lambda)\right)^{-2-n+j} .
\end{aligned}
\end{aligned}
$$

Both terms can be found on the right hand side of relation (70) assuming $|\alpha|=n+1$.

Now let $j=\frac{n}{2}$. Then

$$
\begin{aligned}
&\left(\left(E^{\prime}(\xi)+E^{\prime}(\xi, \lambda)\right)^{0}\left(\lambda \mathbb{I}_{d}+E(\xi)+E(\xi, \lambda)\right)^{-1-\frac{n}{2}}\right)^{\prime} \\
&=\left(E^{\prime}(\xi)+E^{\prime}(\xi, \lambda)\right)\left(\lambda \mathbb{I}_{d}+E(\xi)+E(\xi, \lambda)\right)^{-2-\frac{n}{2}} \\
& \quad=\left(E^{\prime}(\xi)+E^{\prime}(\xi, \lambda)\right)\left(\lambda \mathbb{I}_{d}+E(\xi)+E(\xi, \lambda)\right)^{-1-(n+1)-\left\lfloor\frac{n+1}{2}\right\rfloor}
\end{aligned}
$$

and this term is also on the right hand side of (70) assuming $|\alpha|=n+1$.

We use this lemma to infer

$$
\left|\partial_{\xi}^{\alpha}\left(\lambda \mathbb{I}_{d}+E(\xi)+E(\xi, \lambda)\right)^{-1}\right| \leq c^{\alpha}\left(|\lambda|^{1 / 2}+|\xi|\right)^{-2-|\alpha|} .
$$

An easy calculation yield the validity of (68).

Proof of Theorem 3.3. Recall that, according to the standard decomposition of unity, for all $\varepsilon>0$ there is a covering $\left\{B_{j}\right\}_{j=1}^{\infty}$ of the torus $\Omega$ such that

- $\bigcup_{j=1}^{\infty} B_{j} \supset \Omega$.

- There are functions $\zeta_{j}$ and $\tilde{\zeta}_{j}$ such that $\operatorname{supp} \zeta_{j} \subset B_{j}, \tilde{\zeta}_{j}=1$ on $\operatorname{supp} \zeta_{j}, \operatorname{supp} \tilde{\zeta}_{j} \subset 2 B_{j}$, $\left\|\zeta_{j}\right\|_{W^{2, \infty}}+\left\|\tilde{\zeta}_{j}\right\|_{W^{2, \infty}} \leq c$ with $c$ independent of $j$, and $\sum_{j=1}^{\infty} \zeta_{j}=1$ on $\Omega$.

- Since $\nabla u_{0}, \theta_{0} \in B U C$, we may assume the following property: for every $j \in \mathbb{N}$

$$
\left|\mathbb{D} u_{0}(x)-\mathbb{D} u_{0}\left(x_{j}\right)\right|+\left|\theta_{0}(x)-\theta_{0}\left(x_{j}\right)\right|<\varepsilon
$$

for all $x \in B_{j}$. Here $x_{j}$ is the center of a ball $B_{j}$.

We emphasize that $\varepsilon>0$ will be chosen later. 
For every $j$ we solve an equation

$$
\begin{aligned}
& \lambda u_{j}-\frac{1}{\rho_{*}+\theta_{0}\left(x_{j}\right)} \mathcal{A}\left(\mathbb{D} u_{0}\left(x_{j}\right)\right)(\mathbb{D} u)-\pi^{\prime}\left(\varrho_{*}+\theta_{0}\left(x_{j}\right)\right) \frac{1}{\lambda} \nabla \operatorname{div} u_{j} \\
& =\tilde{\zeta}_{j}\left(F+\left(\frac{1}{\varrho_{*}+\theta_{0}(x)} \mathcal{A}(\mathbb{D} u(x))(\mathbb{D} u)-\frac{1}{\varrho_{*}+\theta_{0}\left(x_{j}\right)} \mathcal{A}\left(\mathbb{D} u_{0}\left(x_{j}\right)\right)(\mathbb{D} u)\right)\right. \\
& \left.+\left(\pi^{\prime}\left(\varrho_{*}+\theta_{0}(x)\right) \frac{1}{\lambda} \nabla \operatorname{div} u_{j}\right)-\pi^{\prime}\left(\varrho_{*}+\theta_{0}\left(x_{j}\right)\right) \frac{1}{\lambda} \nabla \operatorname{div} u_{j}\right) .
\end{aligned}
$$

Since $\mathbb{D} u$ and $\theta$ are bounded and uniformly continuous function, (71) can be written as

$$
\lambda u_{j}-\mathcal{B}_{\lambda} u_{j}=\tilde{\zeta}_{j} F+\tilde{\zeta}_{j} \mathcal{P}_{\lambda j} u_{j}
$$

where $\mathcal{P}_{\lambda j}: W^{2, q}\left(\mathbb{R}^{d}\right) \mapsto L^{q}\left(\mathbb{R}^{d}\right)$ is a linear operator bounded independently of $j$. Note also that $\mathcal{B}_{\lambda}+\mathcal{P}_{\lambda j}=\mathcal{B}_{\lambda}$ on $B_{j}$.

We claim that $\lambda\left(\lambda \mathbb{I}_{d}-\mathcal{B}_{\lambda}-\mathcal{P}_{\lambda j}\right)^{-1},|\lambda|^{\frac{1}{2}} \partial_{k}\left(\lambda \mathbb{I}_{d}-\mathcal{B}_{\lambda}-\mathcal{P}_{\lambda j}\right)^{-1}$ and $\partial_{k} \partial_{l}\left(\lambda \mathbb{I}_{d}-\mathcal{B}_{\lambda}-\mathcal{P}_{\lambda j}\right)^{-1}$ are $\mathcal{R}$-bounded on $\Sigma_{\beta, \nu}$ assuming $\varepsilon$ is small enough. To justify this claim it is enough to repeat the proof of [12, Proposition 4.2]. First, recall that $\mathcal{P}_{\lambda}$ is $\mathcal{R}$-bounded on $\Sigma_{\beta, \nu}$ by ce according to Proposition 4.1. The same proposition also yields $\left(\lambda \mathbb{I}_{d}-\mathcal{B}_{\lambda}\right)^{-1}$ is $\mathcal{R}$-bounded as $\lambda\left(\lambda \mathbb{I}_{d}-\mathcal{B}_{\lambda}\right)^{-1}$ is $\mathcal{R}$-bounded on $\Sigma_{\beta, \nu}$ by Theorem 3.4. We have

$$
\begin{aligned}
\lambda\left(\lambda \mathbb{I}_{d}-\mathcal{B}_{\lambda}-\mathcal{P}_{\lambda j}\right)^{-1}=\lambda\left(\lambda \mathbb{I}_{d}-\mathcal{B}_{\lambda}\right)^{-1}\left(\mathbb{I}_{d}-\mathcal{P}_{\lambda j}\left(\lambda \mathbb{I}_{d}-\right.\right. & \left.\left.\mathcal{B}_{\lambda}\right)^{-1}\right)^{-1} \\
& =\lambda\left(\lambda \mathbb{I}_{d}-\mathcal{B}_{\lambda}\right)^{-1} \sum_{n=0}^{\infty}\left(\mathcal{P}_{\lambda j}\left(\lambda \mathbb{I}_{d}-\mathcal{B}_{\lambda}\right)^{-1}\right)^{n}
\end{aligned}
$$

We use Proposition 4.2 to deduce

$$
\mathcal{R}\left\{\lambda\left(\lambda \mathbb{I}_{d}-\mathcal{B}_{\lambda}\right)^{-1}\left(\mathcal{P}_{\lambda j}\left(\lambda \mathbb{I}_{d}-\mathcal{B}_{\lambda}\right)^{-1}\right)^{n}\right\} \leq \mathcal{R}\left\{\lambda\left(\lambda \mathbb{I}_{d}-\mathcal{B}_{\lambda}\right)^{-1}\right\}\left(c \varepsilon \mathcal{R}\left\{\left(\lambda \mathbb{I}_{d}-\mathcal{B}_{\lambda}\right)^{-1}\right\}\right)^{n}
$$

Now it is enough to take $\varepsilon$ such that $\gamma:=c \varepsilon \mathcal{R}\left\{\left(\lambda \mathbb{I}_{d}-\mathcal{B}_{\lambda}\right)^{-1}\right\}$ is less than 1 . This allows to claim that

$$
\mathcal{R}\left\{\lambda\left(\lambda \mathbb{I}_{d}-\mathcal{B}_{\lambda}-\mathcal{P}_{\lambda j}\right)^{-1}\right\} \leq \mathcal{R}\left\{\lambda\left(\lambda \mathbb{I}_{d}-\mathcal{B}_{\lambda}\right)^{-1}\right\} \frac{1}{1-\gamma}
$$

The same true can be deduced also for the remaining two operators.

Let $\mathcal{T}_{j}(\lambda)$ be a solution operator to (72). We define

$$
\mathcal{W}(\lambda)(f)=\sum_{j=1}^{\infty} \zeta_{j} \mathcal{T}_{j}(\lambda)\left(\tilde{\zeta}_{j} f\right)
$$

Then $u=\mathcal{W}(\lambda)(f)$ fulfills

$$
\lambda u-\mathcal{B}_{\lambda} u=f-\mathcal{K}_{\lambda}(f)
$$

where $\mathcal{K}_{\lambda}: L^{q}(\Omega) \mapsto L^{q}(\Omega)$ is defined as $\mathcal{K}_{\lambda}(f)=\sum_{j=1}^{\infty} \mathcal{B}_{\lambda}\left(\zeta_{j} u_{j}\right)-\zeta_{j} \mathcal{B}_{\lambda}\left(u_{j}\right)$ and $u_{j}=\mathcal{T}_{j}(\lambda)\left(\tilde{\zeta}_{j} f\right)$.

The operator $\mathcal{K}_{\lambda}$ may be written as

$$
\begin{aligned}
\mathcal{K}_{\lambda}=\frac{1}{\varrho_{*}+\theta_{0}} \sum_{j=1}^{\infty} \sum_{k, l, n=1}^{d} a_{m n}^{k l}\left(\mathbb{D} u_{0}\right)\left(\partial_{k} \partial_{l} \zeta_{j} u_{j, n}+\partial_{l} \zeta_{j} \partial_{k} u_{j, n}+\partial_{k} \zeta_{j} \partial_{l} u_{j, n}\right) & \\
& +\sum_{j=1}^{\infty} \pi^{\prime}\left(\varrho_{*}+\theta_{0}\right) \frac{1}{\lambda}\left(\nabla \zeta_{j} \operatorname{div} u_{j}+\nabla^{2} \zeta_{j} u_{j}+\nabla \zeta_{j} \nabla u_{j}\right)
\end{aligned}
$$


and $\mathcal{K}_{\lambda}$ is in particular $\mathcal{R}$-bounded on $\Sigma_{\beta, \nu^{\prime}}$. Indeed, we show this particular boundedness of one term of the right hand side of (173) since the others might be treated in the same way.

$$
\begin{gathered}
\int_{0}^{1}\left\|\sum_{l=1}^{n} r_{l}(v)\left(\sum_{j=1}^{\infty}\left(\nabla \zeta_{j}\right) \operatorname{div} \mathcal{T}_{j}\left(\lambda_{l}\right)\left(\tilde{\zeta}_{j} f\right) \frac{\pi^{\prime}\left(\varrho_{*}+\theta_{0}\right)}{\lambda}\right)\right\|_{L^{q}(\Omega)}^{q} \mathrm{~d} v \\
\quad \leq c \sum_{j=1}^{\infty} \int_{0}^{1}\left\|\sum_{l=1}^{n} r_{l}(v)\left(\nabla \zeta_{j}\right) \operatorname{div} \mathcal{T}_{j}\left(\lambda_{l}\right)\left(\tilde{\zeta}_{j} f\right)\right\|_{L^{q}\left(B_{j} \cap \Omega\right)}^{q} \mathrm{~d} v \\
\leq c \sum_{j=1}^{\infty} \int_{0}^{1}\left\|\sum_{l=1}^{n} r_{l}(v)\left|\lambda_{l}\right|^{-1 / 2}\left|\lambda_{l}\right|^{1 / 2} \operatorname{div} \mathcal{T}_{j}\left(\lambda_{l}\right)\left(\tilde{\zeta}_{j} f\right)\right\|_{L^{q}\left(B_{j} \cap \Omega\right)}^{q} \mathrm{~d} v \\
\leq c \nu^{\prime-1 / 2} \sum_{j=1}^{\infty} \int_{0}^{1}\left\|\sum_{l=1}^{n} r_{l}(v)\left|\lambda_{l}\right|^{1 / 2} \operatorname{div} \mathcal{T}_{j}\left(\lambda_{l}\right)\left(\tilde{\zeta}_{j} f\right)\right\|_{L^{q}\left(B_{j} \cap \Omega\right)} \mathrm{d} v \\
\leq c \nu^{\prime-1 / 2} \sum_{j=1}^{\infty} \int_{0}^{1}\left\|\sum_{l=1}^{n} r_{l}(v) f\right\|_{L^{q}\left(B_{j} \cap \Omega\right)}
\end{gathered}
$$

Consequently, the $\mathcal{R}$-bound of $\mathcal{K}_{\lambda}$ is $c \nu^{\prime-1 / 2}$ and by a proper choice of $\nu^{\prime}$ it is less or equal than $\frac{1}{2}$. We deduce that

$$
\mathcal{R}_{\mathcal{L}\left(L^{q}\right)}\left\{\left(\mathbb{I}_{d}-\mathcal{K}_{\lambda}\right)^{-1}, \lambda \in \Sigma_{\beta, \nu^{\prime}}\right\} \leq 2
$$

See also Proposition 4.2. We thus have $u=\mathcal{W} \circ\left(\mathbb{I}_{d}-\mathcal{K}_{\lambda}\right)^{-1}(f)$ and this solution operator fulfills all the demanded properties.

Note also that the solution to (66) is unique. This comes from the ellipticity of operator $-\mathcal{B}_{\lambda}$.

Proof of Theorem 3.1. Due to Theorem 3.3 we have

$$
\lambda\left(\lambda \mathbb{I}_{d}-\mathcal{A}\right)^{-1} \mathcal{R}-\text { bounded for all } \lambda \in \Sigma_{\beta, \nu}
$$

Especially, $\lambda\left(\lambda \mathbb{I}_{d}-\mathcal{A}\right)^{-1}$ is bounded and by the Hille-Yosida theorem (see [3, Theorem 3.1, Chapter $1]$ ) there exists a semigroup of class $C^{0}$ generated by $\mathcal{A}$. We denote this semigroup $\mathcal{T}$, i.e.

$$
\partial_{t} \mathcal{T}-\mathcal{A T}=0
$$

We set $U(t)=\int_{0}^{t} \mathcal{T}(t-s) F(s)$ d $s$ where $F=(\mathcal{G}, \mathcal{F})$ and we immediately get

$$
\|U\|_{L^{p}\left(0, T ;\left(W^{1, q}(\Omega) \times L^{q}(\Omega)\right)\right)} \leq c\|F\|_{L^{p}\left(0, T ; W^{1, q}(\Omega) \times L^{q}(\Omega)\right)}
$$

where $c$ depends also on the time interval $(0, T)$. Recall that $U$ has two components and we denote them by $\theta$ and $u$. Moreover $U$ solves

$$
\partial_{t} U-\mathcal{A} U=F
$$

which is another form of (64).

Clearly, $U$ solves also

$$
\partial_{t} U+2 \nu U-\mathcal{A} U=F+2 \nu U
$$


We set $G=F+2 \nu U$ and we extend the definition of $G$ and $U$ in such a way that $U(t)=0$, $G(t)=0$ for all $t<0$.

We may write

$$
U(t)=\int_{-\infty}^{\infty} e^{\left(-2 \nu \mathbb{I}_{d}+\mathcal{A}\right)(t-s)} \chi_{[0, \infty)}(t-s) G(s) \mathrm{d} s=\left(e^{\left(-2 \nu \mathbb{I}_{d}+\mathcal{A}\right)(s)} \chi_{[0, \infty)}(s)\right) *(G(s))(t) .
$$

By rules for Fourier transform we get

$$
\widehat{\partial_{t} U}=i \xi\left((-i \xi-2 \nu) \mathbb{I}_{d}+\mathcal{A}\right)^{-1} \widehat{G}
$$

and $M(\xi)=i \xi\left((-i \xi-2 \nu) \mathbb{I}_{d}+\mathcal{A}\right)^{-1}$ is the corresponding multiplier as mentioned in Theorem 3.2. Indeed, using (74), Proposition 4.1 and Proposition 4.2 we infer that $M(\xi)$ and $\xi M^{\prime}(\xi)$ are $\mathcal{R}$-bounded and Theorem 3.2 implies

$$
\left\|\partial_{t} U\right\|_{L^{p}\left(0, T ; W^{1, q}(\Omega) \times L^{q}(\Omega)\right)} \leq c\|G\|_{L^{p}\left(0, T ; W^{1, q}(\Omega) \times L^{q}(\Omega)\right)} \leq c\|F\|_{L^{p}\left(0, T ;\left(W^{1, q}(\Omega) \times L^{q}(\Omega)\right)\right)}
$$

having (75) in mind. Consequently,

$$
\|\theta\|_{W^{1, p}\left(0, T ; W^{1, q}(\Omega)\right)}+\|u\|_{W^{1, p}\left(0, T ; L^{q}(\Omega)\right)} \leq c\left(\|\mathcal{G}\|_{L^{p}\left(0, T ; W^{1, q}(\Omega)\right)}+\|\mathcal{F}\|_{L^{p}\left(0, T ; W^{1, q}(\Omega)\right)}\right) .
$$

Note also that $\theta, u$ solves

$$
2 \nu\left(\begin{array}{l}
\theta \\
u
\end{array}\right)-\mathcal{A}\left(\begin{array}{l}
\theta \\
u
\end{array}\right)=\left(\begin{array}{l}
\mathcal{G} \\
\mathcal{F}
\end{array}\right)-\partial_{t}\left(\begin{array}{l}
\theta \\
u
\end{array}\right)+2 \nu\left(\begin{array}{l}
\theta \\
u
\end{array}\right) .
$$

Theorem 3.3 yields that $\partial_{j} \partial_{k} \lambda^{-1} \mathcal{R}_{\lambda 2}$ is bounded on $\Sigma_{\beta, \nu}$ for every $j, k=1, \ldots, d$ and thus we deduce (with help of (지))

$$
\|u\|_{L^{p}\left(W^{2, q}\right)} \leq c\left(\|\mathcal{G}\|_{L^{p}\left(W^{1, q}\right)}+\|\mathcal{F}\|_{L^{p}\left(W^{1, q}\right)}\right) .
$$

This completes the proof.

\section{Appendix}

Here we present several theorems from other sources for readers convenience.

Theorem 4.1. [Theorem 3.3 in [13]] Let $1<q<\infty$ and let $\Lambda \subset \mathbb{C}$. Let $m(\lambda, \xi)$ be a function defined on $\Lambda \times\left(\mathbb{R}^{d} \backslash\{0\}\right)$ such that for any multi-index $\alpha \in \mathbb{N}_{0}^{d}$ there exists a constant $C_{\alpha}$ depending on $\alpha$ and $\Lambda$ such that

$$
\left|\partial_{\xi}^{\alpha} m(\lambda, \xi)\right| \leq C_{\alpha}|\xi|^{-\alpha}
$$

for any $(\lambda, \xi) \in \Lambda \times\left(\mathbb{R}^{d} \backslash\{0\}\right)$. Let $K_{\lambda}$ be an operator defined by $K_{\lambda} f=\mathcal{F}_{\xi}^{-1}[m(\lambda, \xi) \hat{f}(\xi)]$. Then, the set $\left\{K_{\lambda} \mid \lambda \in \Lambda\right\}$ is $\mathcal{R}$-bounded on $\mathcal{L}\left(L^{q}\left(\mathbb{R}^{d}\right)\right)$ and

$$
\mathcal{R}_{\mathcal{L}\left(L^{q}\left(\mathbb{R}^{d}\right)\right)}\left(\left\{K_{\lambda} \mid \lambda \in \Lambda\right\}\right) \leq C_{q, d} \max _{|\alpha| \leq d} C_{\alpha}
$$

Lemma 4.1 (Lemma 3.1 in [13]). Let $0<\beta<\pi / 2$ and $\nu_{0}>0$. For any $\lambda \in \Sigma_{\beta, \nu}$ we have

$$
\left.|\lambda+| \xi\right|^{2} \mid \geq \sin (\beta / 2)\left(|\lambda|+|\xi|^{2}\right) .
$$


Proposition 4.1 (Proposition 2.13 in [13]). Let $D \subset \mathbb{R}^{d}$ be a domain and let $\Lambda$ be a domain in $\mathbb{C}$. Let $m(\lambda)$ be a bounded function on $\Lambda$ and let $M_{m}(\lambda): L^{q}(D) \mapsto L^{q}(D)$ be defined as $M_{m}(\lambda) f=m(\lambda) f$. Then

$$
\mathcal{R}_{\mathcal{L}\left(L^{q}(D)\right)}\left(\left\{M_{m}(\lambda) \mid \lambda \in \Lambda\right\}\right) \leq C_{d, q, D}\|m\|_{L^{\infty}(\Lambda)}
$$

Proposition 4.2 (Proposition 2.16 in [13] or Proposition 3.4 in [12]). 1. Let $X$ and $Y$ be $B a$ nach spaces and let $\mathcal{T}$ and $\mathcal{S}$ be $\mathcal{R}$-bounded families on $\mathcal{L}(X, Y)$. Then $\mathcal{T}+\mathcal{S}=\{T+S \mid T \in$ $\mathcal{T}, S \in \mathcal{S}\}$ is also $\mathcal{R}$-bounded on $\mathcal{L}(X, Y)$ and

$$
\mathcal{R}_{\mathcal{L}(X, Y)}(\mathcal{T}+\mathcal{S}) \leq \mathcal{R}_{\mathcal{L}(X, Y)}(\mathcal{T})+R_{\mathcal{L}(X, Y)}(\mathcal{S})
$$

2. Let $X, Y$ and $Z$ be Banach spaces and let $\mathcal{T}$ and $\mathcal{S}$ be $\mathcal{R}$-bounded families on $\mathcal{L}(X, Y)$ and $\mathcal{L}(Y, Z)$ respectively. Then $\mathcal{S} \mathcal{T}=\{S T \mid T \in \mathcal{T}, S \in \mathcal{S}\}$ is $\mathcal{R}$-bounded on $\mathcal{L}(X, Y)$ and

$$
\mathcal{R}_{\mathcal{L}(X, Z)}(\mathcal{S} \mathcal{T}) \leq \mathcal{R}_{\mathcal{L}(X, Y)}(\mathcal{T}) \mathcal{R}_{\mathcal{L}(Y, Z)}(\mathcal{S})
$$

Acknowledgement: The research of Š.N. and V.M. leading to these results has received funding from the Czech Sciences Foundation (GAČR), GA19-04243S and in the framework of RVO: 67985840. The research of M.K. was supported by RVO: 67985840.

\section{References}

[1] Anna Abbatiello, Eduard Feireisl, and Antonín Novotný. Generalized solutions to models of compressible viscous fluids. arXiv:1912.12896.

[2] Herbert Amann. Linear and quasilinear parabolic problems. Vol. II, volume 106 of Monographs in Mathematics. Birkhäuser/Springer, Cham, 2019. Function spaces.

[3] Viorel Barbu. Nonlinear semigroups and differential equations in Banach spaces. Editura Academiei Republicii Socialiste România, Bucharest; Noordhoff International Publishing, Leiden, 1976. Translated from the Romanian.

[4] Hamid Bellout and Frederick Bloom. Incompressible bipolar and non-Newtonian viscous fluid flow. Advances in Mathematical Fluid Mechanics. Birkhäuser/Springer, Cham, 2014.

[5] Hamid Bellout, Frederick Bloom, and Jindřich Nečas. Solutions for incompressible nonNewtonian fluids. C. R. Acad. Sci. Paris Sér. I Math., 317(8):795-800, 1993.

[6] Hamid Bellout, Frederick Bloom, and Jindřich Nečas. Young measure-valued solutions for non-Newtonian incompressible fluids. Comm. Partial Differential Equations, 19(11-12):17631803, 1994.

[7] Dieter Bothe and Jan Prüss. $L_{P}$-theory for a class of non-Newtonian fluids. SIAM J. Math. Anal., 39(2):379-421, 2007.

[8] Donald L. Burkholder. Martingales and Fourier analysis in Banach spaces. In Probability and analysis (Varenna, 1985), volume 1206 of Lecture Notes in Math., pages 61-108. Springer, Berlin, 1986. 
[9] Frédéric Charve and Raphaël Danchin. A global existence result for the compressible NavierStokes equations in the critical $L^{p}$ framework. Arch. Ration. Mech. Anal., 198(1):233-271, 2010 .

[10] Philippe Clément and Jan Prüss. An operator-valued transference principle and maximal regularity on vector-valued $L_{p}$-spaces, volume 215 of Lecture Notes in Pure and Appl. Math. Dekker, New York, 2001.

[11] Giuseppe Da Prato and Pierre Grisvard. Sommes d'opérateurs linéaires et équations différentielles opérationnelles. J. Math. Pures Appl. (9), 54(3):305-387, 1975.

[12] Robert Denk, Matthias Hieber, and Jan Prüss. R-boundedness, Fourier multipliers and problems of elliptic and parabolic type. Mem. Amer. Math. Soc., 166(788):viii+114, 2003.

[13] Yuko Enomoto and Yoshihiro Shibata. On the R-sectoriality and the initial boundary value problem for the viscous compressible fluid flow. Funkcial. Ekvac., 56(3):441-505, 2013.

[14] Eduard Feireisl, Antonín Novotný, and Hana Petzeltová. On the existence of globally defined weak solutions to the Navier-Stokes equations. J. Math. Fluid Mech., 3(4):358-392, 2001.

[15] Dario Graffi. Il teorema di unicità nella dinamica dei fluidi compressibili. J. Rational Mech. Anal., 2:99-106, 1953.

[16] Nobutoshi Itaya. On the cauchy problem for the system of fundamental equations describing the movement of compressible viscous fluid. Ködai Math. Sem. Rep., 23:60-120, 1971.

[17] Nobutoshi Itaya. On the initial value problem of the motion of compressible viscous fluid, especially on the problem of uniqueness. J. Math. Kyoto Univ., 16(2):413-427, 1976.

[18] Peer C. Kunstmann and Lutz Weis. Maximal $L_{p}$-regularity for parabolic equations, Fourier multiplier theorems and $H^{\infty}$-functional calculus. In Functional analytic methods for evolution equations, volume 1855 of Lecture Notes in Math., pages 65-311. Springer, Berlin, 2004.

[19] Olga A. Ladyženskaja, Vsevolod A. Solonnikov, and Nina N. Uralceva. Linear and quasilinear equations of parabolic type. Translated from the Russian by S. Smith. Translations of Mathematical Monographs, Vol. 23. American Mathematical Society, Providence, R.I., 1968.

[20] Olga A. Ladyzhenskaya. The mathematical theory of viscous incompressible flow. Revised English edition. Translated from the Russian by Richard A. Silverman. Gordon and Breach Science Publishers, New York-London, 1963.

[21] Pierre-Louis Lions. Mathematical topics in fluid mechanics. Vol. 2, volume 10 of Oxford Lecture Series in Mathematics and its Applications. The Clarendon Press, Oxford University Press, New York, 1998. Compressible models, Oxford Science Publications.

[22] Josef Málek, Jindřich Nečas, Mirko Rokyta, and Michael Rưžička. Weak and measure-valued solutions to evolutionary PDEs, volume 13 of Applied Mathematics and Mathematical Computation. Chapman \& Hall, London, 1996.

[23] Josef Málek, Jindřich Nečas, and Michael Růžička. On the non-Newtonian incompressible fluids. Math. Models Methods Appl. Sci., 3(1):35-63, 1993. 
[24] Alexander E. Mamontov. On the global solvability of the multidimensional Navier-Stokes equations of a nonlinearly viscous fluid. I. Sibirsk. Mat. Zh., 40(2):408-420, iii, 1999.

[25] Alexander E. Mamontov. On the global solvability of the multidimensional Navier-Stokes equations of a nonlinearly viscous fluid. II. Sibirsk. Mat. Zh., 40(3):635-649, iii, 1999.

[26] Akitaka Matsumura and Takaaki Nishida. The initial value problem for the equations of motion of viscous and heat-conductive gases. J. Math. Kyoto Univ., 20(1):67-104, 1980.

[27] Akitaka Matsumura and Takaaki Nishida. Initial-boundary value problems for the equations of motion of compressible viscous and heat-conductive fluids. Comm. Math. Phys., 89(4):445464, 1983.

[28] Šárka Matušů-Nečasová and Antonín Novotný. Measure-valued solution for non-Newtonian compressible isothermal monopolar fluid. Acta Appl. Math., 37(1-2):109-128, 1994. Mathematical problems for Navier-Stokes equations (Centro, 1993).

[29] John Nash. Le problème de Cauchy pour les équations différentielles d'un fluide général. Bull. Soc. Math. France, 90:487-497, 1962.

[30] Jan Prüss. Maximal regularity for evolution equations in $L_{p}$-spaces. Conf. Semin. Mat. Univ. Bari, (285):1-39 (2003), 2002.

[31] José L. Rubio de Francia. Martingale and integral transforms of Banach space valued functions. In Probability and Banach spaces (Zaragoza, 1985), volume 1221 of Lecture Notes in Math., pages 195-222. Springer, Berlin, 1986.

[32] James Serrin. On the uniqueness of compressible fluid motions. Arch. Rational Mech. Anal., 3:271-288 (1959), 1959.

[33] Yoshihiro Shibata and Senjo Shimizu. On the maximal $L_{p} L_{q}$ regularity of the Stokes problem with first order boundary condition; model problems. J. Math. Soc. Japan, 64(2):561-626, 2012 .

[34] Vsevolod A. Solonnikov. The solvability of the initial-boundary value problem for the equations of motion of a viscous compressible fluid. Zap. Naučn. Sem. Leningrad. Otdel. Mat. Inst. Steklov. (LOMI), 56:128-142, 197, 1976. Investigations on linear operators and theory of functions, VI.

[35] Hiroki Tanabe. Functional analytic methods for partial differential equations, volume 204 of Monographs and Textbooks in Pure and Applied Mathematics. Marcel Dekker, Inc., New York, 1997.

[36] Alberto Valli. Periodic and stationary solutions for compressible Navier-Stokes equations via a stability method. Ann. Scuola Norm. Sup. Pisa Cl. Sci. (4), 10(4):607-647, 1983.

[37] Alberto Valli and Wojciech M. Zajaczkowski. Navier-stokes equations for compressible fluids: global existence and qualitative properties of the solutions in the general case. Comm. Math. Phys., 103(2):259-296, 1986.

[38] Aizik I. Vol'pert and Sergei I. Hudjaev. The Cauchy problem for composite systems of nonlinear differential equations. Mat. Sb. (N.S.), 87(129):504-528, 1972. 
[39] Lutz Weis. Operator-valued Fourier multiplier theorems and maximal $L_{p}$-regularity. Math. Ann., 319(4):735-758, 2001. 